\title{
1 Sumo-mediated recruitment allows timely function of the Yen1 2 nuclease in mitotic cells
}

4 Hugo Dorison ${ }^{1,3}$, Ibtissam Talhaoui ${ }^{1}$ and Gerard Mazón ${ }^{1,2^{*}}$

1 Université Paris-Saclay, UMR9019 CNRS, Gustave Roussy. 114, rue Édouard Vaillant, 94800 Villejuif, France.

${ }^{2}$ Inserm - Institut National de la Santé et de la Recherche Médicale, France.

3 Present Address: Hôpital Henri-Mondor, IMRB- U955 Inserm, 51 Av Maréchal De Lattre Tassigny, 94010 Créteil, France.

$6 \quad$ *Corresponding author: Gerard Mazón.

$7 \quad$ Email gerard.mazon@gustaveroussy.fr

9 Author Contributions: GM obtained funding, designed experiments, contributed to Figures 1, 2, $106, \mathrm{~S} 1, \mathrm{~S} 3, \mathrm{~S} 4$ and wrote the manuscript. HD edited the manuscript and performed experiments for 11 Figures 1, 2, 4, 5, 6, S1, S2 and S5. IT edited the manuscript and performed experiments for 12 figures $1,3, \mathrm{~S} 3$ and $\mathrm{S} 4$.

13 Competing Interest Statement: No competing interests.

14 Keywords: Homologous Recombination. Structure-selective endonucleases. Sumoylation.

15 The Manuscript consist in a PDF file including:

$16 \quad$ Main Text

$17 \quad$ Figures 1 to 5

18 Supplementary information includes 5 figures and 11 tables that can be found in a separate document. 


\section{Abstract}

22 The modification of DNA damage response proteins with Sumo is an important mechanism to

23 orchestrate a timely and orderly recruitment of repair factors to damaged sites. After replication

24 stress and double-strand break formation a number of repair factors are Sumoylated and interact

25 with other Sumoylated factors, including the nuclease Yen1. Yen1 plays a critical role to ensure

26 genome stability and unperturbed chromosome segregation by removing covalently linked DNA

27 intermediates that are formed by homologous recombination. Here we show how this important

28 role of Yen1 is dependent on interactions mediated by non-covalent binding to Sumoylated

29 partners. Mutations in the motifs that allow Sumo-mediated recruitment of Yen1 impair its ability

30 to resolve DNA intermediates and result in increased genome instability and chromosome mis-

31 segregation. 
Introduction

The DNA integrity of the genomes is constantly exposed to multiple challenges either from endogenous or exogenous sources of DNA damage. Cells have evolved multiple DNA repair pathways to ensure genome stability, Homologous Recombination (HR) being one of the most critical pathways to specifically counter the deleterious DNA double-strand breaks and other problems in the DNA integrity arising during replication. As the HR pathway operates, different DNA substrates and intermediates are formed that physically inter-connect distinct DNA molecules creating a joint-molecule (JM) intermediate. These intermediates are a threat to the successful segregation of chromosomes and are to be dismantled during mitosis by different specialized proteins acting in concert to prevent segregation defects and genome rearrangements. In yeast, the dissolution pathway mediated by the complex of Sgs1-Top3-Rmi1 (STR) ensures the disentanglement and release of double Holliday Junctions (dHJs) and two other helicases, Mph1 and Srs2, act early on the pathway preferentially over D-loop intermediates to reduce the number of JM intermediates and ensure the completion of the recombinational repair without crossing-over between the involved DNA templates. Opposed to these noncrossover (NCO) pathways, the nucleolytic processing of these JM intermediates can result in reciprocal crossovers (COs), with the risk of genome rearrangements and loss of heterozygosity (LOH) events $(1,2)$.

Given the risk for genome stability of a nucleolytic processing of HR intermediates, the different actors able to cleave these intermediates are strictly controlled and used as an option of last resort in DNA substrates not previously dismantled by the action of helicases $(3,4)$. Two major nucleases are involved in the nucleolytic processing of recombination intermediates in the yeast model, Mus81-Mms4 and Yen1 (5). The Mus81-Mms4 nuclease plays different roles at replication forks, and is gradually hyper-activated by Cdc5- and Cdc28/CDK1-dependent phosphorylation of Mms4 to peak its activity in late G2/M (6-8) where it associates with the Slx4-Dpb11 scaffold (9). Its broad substrate recognition enables Mus81-Mms4 to cleave 3'-flap containing DNA substrates and HJs, preferentially when they are still nicked or not completely ligated (10). Its hyperactivation in late G2 and its broad substrate specificity positions Mus81-Mms4 in a critical role to cleave both captured D-loop and early $\mathrm{HJ}$ intermediates, possibly targeting these intermediates that can't complete full conversion to a $\mathrm{dHJ}$ and thus remain inaccessible to processing by the STR complex (3). As mitosis progresses, the Cdc14 phosphatase will trigger the reversal of the inhibitory Cdc28-mediated phosphorylation of Yen1 in turn allowing its nuclear localization and its proper substrate recognition $(11,12)$. This late activation of Yen1 at the anaphase entry ensures

66 that all remaining recombination intermediates, especially those that escaped dissolution by STR 67 or cleavage by Mus81-Mms4, are resolved before mitotic exit $(11,12)$. To ensure the clearing of 
68 Yen1 nuclease from the nucleus in the subsequent S-phase, and prevent off-targeted activity directed to 5'-flap containing DNA intermediates, Yen1 is additionally controlled by a Sumotargeted degradation mediated by the SIx5-SIx8 ubiquitin ligase, further limiting the potential of crossover formation (13).

72 Protein covalent modification with the small ubiquitin-like modifier (Sumo) (14) is an important 73 mechanism to fine tune DNA-mediated transactions during the DNA damage and repair 74 responses (15-18). In Saccharomyces cerevisiae Sumoylation occurs in a multi-step reaction 75 involving the E1 Aos1-Uba2 activating enzyme dimer, the E2 conjugating enzyme Ubc9, and 76 three possible E3 ligases (Siz1, Siz2 and Mms21), with some redundancy of Siz1 and Siz2 for its 77 substrates (19-21). Several players of the HR pathway, besides the nuclease Yen1, are also 78 found among the Sumoylated DNA repair targets, including Rad52, PCNA, RPA and Sgs1 (17, 22-26). Sumoylation is able to influence biological processes in different ways. Proteins can be mono-Sumoylated, multi-Sumoylated or poly-Sumoylated, and the modification will re-design the protein surfaces allowing changes in protein activity, or in its way it can interact with other proteins. One of the best-described effects of protein Sumoylation is the enabling of interaction with other protein partners in a bait-to-prey fashion using Sumo as the moiety that is recognized by a specific domain in the partner protein, called a Sumo Interacting Motif (SIM). These motifs are found throughout species and according to its amino acid composition can be classified in several families of consensus sequences (27). Most SIMs can be defined as a core stretch of four amino acids with a majority of hydrophobic residues (typically rich in $\mathrm{V} / \mathrm{I} / \mathrm{L}$ ). This hydrophobic core fits into the hydrophobic groove on the Sumo surface and is often flanked by a stretch of 3-4 acidic or polar residues in the SIM sequence that interact with basic residues on the surface of Sumo (28-31). SIM types showing the flanking stretch of acidic residues present thus a similar architecture to that of ubiquitin interacting motifs (UIM) that also show a key stretch of polar residues flanking the hydrophobic core $(32,33)$. SIM motifs are able to interact with mono- or poly-Sumoylated proteins and can be usually present in tandem dispositions, probably helping interact with multiple Sumoylated lysines or with a poly-Sumoylated lysine in the interacting protein $(28,34,35)$. Interactions by Sumo-SIM partnerships are extremely labile and can be easily induced and curbed down by altering the Sumoylation status of the involved proteins. This flexibility allows a quick building of protein complexes in response to changing stress conditions in the cell $(17,35)$. The build up of these protein complexes by Sumo-mediated recruitment via SIMs is generally associated to the actual Sumoylation of the two involved proteins $(36,37)$. Then, it was thus not surprising to identify in Yen1, which is Sumoylated (13), several putative Sumo interacting motifs. In the present study we define two functional SIMs in the Yen1 Cterminal region that play important roles in the nuclease sub-nuclear localization and its function 
alleviating the persistence of chromosome-segregation challenging JMs throughout the end of mitosis.

\section{Results} stressed before, Sumoylated proteins are able to transiently interact with a variable strength to other proteins containing SIMs. These motifs consist in a stretch of amino-acids with a core of aliphatic residues, often flanked by three or more amino-acids with a negative charge or susceptible to become phosphorylated (27). We inspected the Yen1 sequence through available algorithms (27) to detect SIM motifs and we identified several interesting hits in the primary sequence of Yen1 (Figure 1A). To validate the presence of such motifs in Yen1 we performed a two-hybrid analysis with Smt3 as bait and either wild type full-length or truncated versions of Yen1 as a prey (Figure 1B). The ability to interact with Sumo in the two-hybrid assay was only retained by the C-terminal part of Yen1 (amino-acids 354 to 759 ) and mutations in two putative SIMs present in that half of the protein completely abolished the interaction. A mutation in the first SIM, a type $r$ motif (27) with a core at amino-acids 636 to 642 and a flanking stretch of acidic residues was indeed sufficient to almost completely impair the interaction to Smt3 in the twohybrid assay (Figure 1B). Interestingly, this motif is highly conserved across Yen1 in other fungi and is very reminiscent of SIMs found in the SIx5, Rad18 and Elg1 proteins $(36,38,39)$ (Figure

\section{$<$ Figure 1>}

While mutation on the consensus SIM sites abolishes the interaction in the two-hybrid assay, this assay was suggested to reflect a covalent modification of Yen1 (40) and thus the effect detected in this test might be an indirect effect reflecting the loss of direct sumoylation of Yen1 related to the absence of non-covalent interaction of Yen1 to SUMO through its SIMs. Moreover, a possible SIM-mediated interaction would be difficult to be confirmed in this test if polymerization of Smt3 is needed or a Sumo covalent modification of a bridging partner is required for the growth read-out of the test. To better confirm the nature of the interaction lost in our mutants and thus validate the SIM sites, we used a pull-down approach (36). GST-Smt3 was over-expressed and purified from bacteria, and bound to a Glutathione resin. Purified Yen1 or its mutant SIM variants were then allowed to bind to the pre-bound GST-Smt3, and after several washes, the column content was eluted in denaturing conditions and inspected by western blotting (Figure 1C). Yen1 was detected

138 in the eluates thus confirming its ability to interact non-covalently to Smt3, but was much less 
retained (30\% compared to wild-type) when bearing mutations in both of its SIMs, while inactivation of only one of the two motifs did not significantly altered retention suggesting that at least in vitro, the presence of one single SIM at the Yen1 C-terminal region confers it ability to bind Smt3 (Figure 1C). While the GST-Smt3 column may indicate the ability of Yen1 to bind to monomers of Smt3 through its SIMs, the packed dispositions of GST-Smt3 in the column can mimick a poly-Sumoylated chain and contact Yen1 simultaneously in multiple SIM sites. To further evaluate the non-covalent binding of Yen1 to poly-Smt3 we decided to generate an affinity column containing poly-Sumo chains to test whether they have the ability to non-covalently bind to Yen1 and thus capture the protein. We generated Smt3 chains by adding Aos1-Uba2 and Ubc9 in a reaction with purified 6xHis-Smt3 (13), the resulting poly-Sumo chains were dialyzed and used to bind to a Cobalt HisPur Superflow agarose matrix, the poly-Smt3 coated matrix was used to test retention of Yen1-1xHA (Figure 1D). While the column retained the wild-type Yen1, the recovery of the protein mutated in both SIMs (Yen1 $1^{\mathrm{SIM} 1-2 \Delta \Delta}$ ) was greatly decreased (Figure $1 \mathrm{E})$, thus confirming that Yen1 binds non-covalently to poly-Sumo chains, a binding that depends on the presence of the two identified Yen1's SIMs.

\section{Strains carrying SIM-defective variants of Yen1 display increased sensitivity to DNA} damage. We next aimed to understand the effect of the mutations in the SIM motifs on the ability of Yen 1 to be normally regulated by Cdk1/Cdc14 and shuttled timely to the nucleus. Given the proximity of Yen1's SIMs to its NLS, a C-terminal GFP fusion of the Yen1 mutants was monitored to see if any gross defect occurred for its nuclear shuttling (Figure 2). Both single and double SIM mutants presented nuclear exclusion in S-phase as the wild-type and were nuclear in late mitosis and G1 and the relative distribution of GFP intensity detected in the nucleus or in the cytoplasm at the different cell cycle phases was not changed between the different Yen1 variants (Figure $2 \mathrm{~B}$, Figure S2). We also analyzed the pattern of cyclic phosphorylation by synchronizing cells in G1 and analysing the mobility of Yen1 at different time points after its release (Figure 2C, Figure $\mathrm{S} 2 \mathrm{~A}$ ). All the mutants displayed a normal cycle of phosphorylation in S-phase followed by gradual de-phosphorylation with only slight variations in the total amount of the protein all across cellcycle phases. Next we asked whether the presence of an endogenous copy of the SIM mutants would compromise the ability of Yen1 to back-up for the functions of Mus81-Mms4 (5). The mutants were introduced into a mus81 $1 \Delta$ background and tested for its sensitivity to an array of DNA damaging agents (Figure $2 \mathrm{D}$ and $\mathrm{E}$ ). Cells with a double mutation mus81 $1 \Delta$ yen $1 \Delta$ are extremely sensitive to MMS at low doses, and they also present a moderate sensitivity to the radiomimetic drug Zeocin and to the replication stalling drug Hydroxyurea $(H U)$ (13), both a mutation in the first SIM and the double SIM mutant significantly increased the sensitivity of a mus81 1 strain to MMS, while increased sensitivity to MMS after mutation in the second SIM 
175 alone was not significant (Figure 2D, 2E, Table S3). Mutation in both SIMs was necessary to see

176 a moderate increase in sensitivity to Zeocin (Figure 2D, 2E). Mutation in both SIMs was also

177 necessary to sensitize cells at $20 \mathrm{mM} \mathrm{HU}$ and while individual SIM mutants sensitized cells to 40

$178 \mathrm{mM} \mathrm{HU}$, at such dose survival was already compromised by a two log difference in cells bearing 179 both SIM mutations or lacking YEN1 (Figure 2E). Yen1 has also been described to be essential in 180 cells with a deletion on the DNA2 helicase-nuclease in the presence of its suppressor pif1 (41181 44). Similarly, cells carrying the dna2-2 helicase-deficient allele rely on the activity of YEN1 to 182 process DNA intermediates in this background (41). Similar to what was already observed in 183 dna2-2 cells in the W303 background (41) we found that a dna2-2 mutation is partially unviable 184 with yen1s (Figure S3). The dna2-2 cells, while viable, present a strong heterogeneity of 185 phenotypes likely associated to spontaneous accumulation of suppressors, as described (41) 186 (Figure S3). Nonetheless, the introduction of the more severe of the SIM alleles, carrying 187 simultaneous mutations in both SIMs, was viable in a dna2-2 background, suggesting a minor 188 role of non-covalent Sumo binding for the Yen1's functions required in a dna2-2 context. In

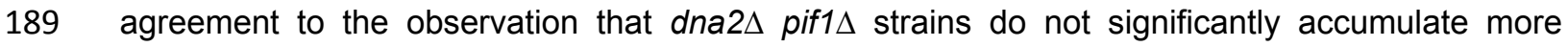

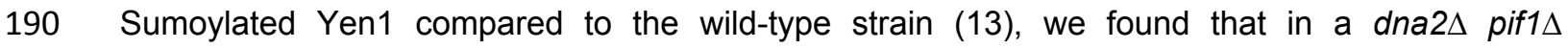
191 background the SIM defective allele does not increase the already strong sensitivity of cells to 192 DNA damaging agents like MMS or HU (Figure S3).

$<$ Figure 2>

Mutation of the SIMs induces a sumo-less Yen1 phenotype in vivo. In other Sumoylated DNA repair proteins containing functional SIMs, the mutation of these motifs has an impact in the ability of the protein to be directly Sumoylated $(36,37)$. The results in the two-hybrid experiments suggested such an effect for Yen1 (Figure 1), which we have previously characterized to be Sumoylated ina Siz1/Siz2-dependent manner (13). To further confirm the absence of covalent sumoylation after impairment of Yen1's SIMs we compared the Sumoylation levels of the wildtype and the SIM-defective Yen1 mutants by performing denaturing pull-downs of His-tagged Smt3 (Figure 3A). Yen1 Sumoylation peaks when cells are exposed to high MMS doses (13) and we reproduced Yen1 Sumoylation in these conditions for the wild-type protein (Figure 3A).

205 Nonetheless, the fraction of Sumoylated Yen1 in the mutant in either the first SIM motif or the 206 double mutant in the two SIM motifs was greatly reduced in conditions with similar input levels to $2075 \%$ and $1 \%$ of the wild-type levels respectively (Figure $3 \mathrm{~A}$ ), mutation of SIM2 had a milder effect 208 reducing the recovery of Sumoylated forms to $\approx 20 \%$ of the forms recovered in the wild-type 209 (Figure 3A). The gradual effects detected for individual or combined mutations of both SIMs 210 points to a concerted action of both motifs to promote Yen1 Sumoylation by allowing Yen1 non- 
211 covalent binding to Smt3. Despite its sensitivity to very low doses of MMS, cells carrying dna2-2

212 did not spontaneously increase the yield of Sumoylated forms of Yen1 while we detected in such

213 conditions under spontaneous damage a minor yield of Sumoylation in the presence of mutations

214 on both Yen1 SIMs in either a wild-type or a dna2-2 background (Figure S3).

215

$216<$ Figure 3>

The mutations introduced to inactivate the SIM sites do not contain any lysine substitution, and SIM1 is not directly flanked by lysines in the immediate vicinity. To further confirm that the lack of Sumoylation was not due to un-adverted absence or un-accessibility of Sumoylation-target lysines in Yen1, we decided to test the mutant proteins in an in vitro Sumoylation reaction. After a reaction of the SIM mutants of Yen1 with Aos1-Uba2 and the conjugating enzyme Ubc9, a normal

223 Sumoylation pattern was detected with the same ladder of bands of increasing sizes for all Yen1

224 variants (Figure 3B). We also performed a complete ligation reaction containing Siz2 as E3,

225 increasing the yield of the reaction. Comparing the ligation reactions over time we couldn't detect

226 significant differences in the amount or timing of accumulation of the Sumoylated forms (Figure

227 3B, Figure S4) that in all the Yen1 variants achieved complete Sumoylation of the substrates at

228 similar time points. We conclude that the presence of the SIM mutations does not preclude

229 modification of any of the Yen1's lysines targeted by the Sumoylation machinery.

230 The c-terminal domain of Yen1 containing the two SIMs is dispensable for a complete nuclease 231 activity (45). Nonetheless, we verified that the mutation of both SIMs does not impair Yen1's 232 nuclease activity in vitro by using a synthetic Holliday Junction (46) as a substrate. Immuno233 precipitated Yen1 was added to cleavage reactions, and we compared the yield of HJ cutting for 234 either the wild-type Yen1 and the SIM defective mutant Yen1 ${ }^{\text {SIM1-2 }}{ }^{2 \Delta}$. The nuclease activity was 235 undistinguishable for both the wild-type and the mutant, that were able to linearize the $\mathrm{HJ}$ 236 substrate at similar rates (Figure 3C). Alteration of the SIM motifs at the C-terminal part of the 237 protein seems thus not to alter the cutting efficiency of Yen1, whose nuclease and conserved 238 XPG domains are present at the $\mathrm{N}$-terminal part of the protein (Figure 1A). Nonetheless, the test 239 did not take into account the disposition of the junction in a chromatin context in the cell that could 240 influence the ability to cut $\mathrm{HJ}$ in vivo.

242 Localization to spontaneous and induced sites of activity is impaired by inactivation of 243 the Yen1's SIMs. Sumoylation and interaction with SIMs has been proposed as a way to enforce 244 a cascade of interactions to foster recruitment of factors to specific subcellular locations (35). We 245 decided to determine if the impairment of the Yen1 SIMs was altering its ability to cluster in foci 246 that are observed to occur either spontaneously or induced by DNA damage $(4,13)$. C-terminal 
247 GFP tagged versions of Yen1 were compared for its foci distribution (Figure 4, Figure S5, Table

248 S4 and S5), the number of cells showing spontaneous foci in a wild-type strain ranges around

$24910 \%$, while only $3 \%$ of the cells in the double SIM mutant displayed foci in strains with a functional

250 Mus81-Mms4 (Figure 4B). The effect was more marked when observing the spontaneous foci in

251 a mus $81 \Delta$ strain, where cells displaying foci decreased from $30 \%$ to $3-4 \%$ in the SIM mutant

252 (Figure 4B). These foci were equally decreased in the presence of exogenous damages,

253 suggesting that Yen1 SIMs are equally important to properly localize the protein to spontaneous

254 damaged sites and exogenous damages sites (Figure 4B, Table S6 and S7).

$256<$ Figure 4>

Absence of Yen1's SIMs prevent accumulation of the Yen1 fraction targeted by SIx5-SIx8.

259 We have demonstrated in a previous work a role for the SIx5-SIx8 Sumo-targeted ubiquitin ligase

260 in the removal of a subset of Yen1 from the nucleus during the transition from $\mathrm{G} 1$ to $S$ phase (13).

261 As a result, cells defective in SIx8 show a persistence of Yen1 foci, which are detected in large

262 numbers even in the presence of functional Mus81-Mms4 (13). We wondered if the absence of

263 proper localization in the SIM mutant would prevent Yen1 accumulation in the absence of SIx5-

264 SIx8. According to our expectations, a deletion of $s / x 8 \Delta$ in the strain bearing the mutations in the

265 Yen1's SIMs did not increase the number of Yen1 foci, thus suggesting mis-localization of Yen1

266 in the mutant strain prevents the need for SIx5-SIx8 targeted removal (Figure 5A, Table S8). The

267 persistence of the SIx5-SIx8 targeted Yen1 fraction can also be detected by performing a

268 cycloheximide chase during a synchronous release from $\mathrm{G} 1$, and observing the degradation of

269 Yen1 under inhibition of de novo protein synthesis in this cell-cycle interval (13). In a s/x84

270 background, the double SIM mutant protein was degraded during such cycloheximide chase

271 faster than the wild-type protein (Figure 5B), indicating that the lack of accumulation of Yen1 at

272 nuclear sites does also dispense for a targeted removal of this subset of the nuclease, that is

273 removed timely even in the absence of Sumo-targeted ubiquitination by SIx5-SIx8.

274

$275<$ Figure 5>

276

277 Unpaired Sumo-directed localization induces an increase in untimely chromosome

278 segregation. The presence of both mus $81 \Delta$ and yen $1 \Delta$ deletions makes cells synergistically

279 sensitive to drugs like MMS, as stated before, and also increase their spontaneous number of 280 chromosome mis-segregations monitored either by dedicated genetic systems $(5,47)$ or by a 281 direct observation of fluorescent-tagged chromosomes during mitotic divisions (13) (Figure 5C).

282 We compared the mitoses of single mus $81 \Delta$ cells to that of cells carrying mus $81 \Delta$ and the allele 


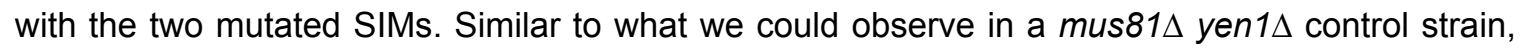
mus81 $\Delta$ cells with the mutated Yen1 SIMs displayed an increased number of segregation issues. As it can be observed in the violin plots displaying the time that individual monitored cells spent to fully segregate the fluorescent tag, the Yen1 double SIM mutant does segregate chromosomes in a similar average time than the wild type for those cells completing full segregation (Figure 5D) but about $30 \%$ of the cells carrying these Yen 1 SIM mutations in the mus $81 \Delta$ background were unable to resolve its segregation within the time of video-microscopy observation (2 h), and were classified as non-disjunctions (Figure 5D, Table S9). The high number of chromosome missegregation detected for the Yen1 mutant variant in a mus81 background is in line with that observed in cells completely lacking Yen1 clearly pointing to a faulty function of Yen1 when SIMs are absent.

\section{Mutation in the SIMs of Yen1 reduces the formation of crossing-over after a single DSB. To} further browse the implications of the presence of a defective Sumo-interacting Yen1 allele for the actual resolution of recombination intermediates, we decided to analyse the level of crossing-over (CO) formation in two widely used tests that estimate the $\mathrm{CO}$ levels after a single DSB formation $(3,5,48)$ (Figure $6 A$ and D). In accordance with the increased sensitivity to different DNA damaging agents observed for the Yen1 allele carrying the SIM mutations when combined with a mus81D background (Figure 2), we detected a decreased formation of crossovers in this genotype after the induction of a single DSB in a diploid tester strain (5), half-way to the

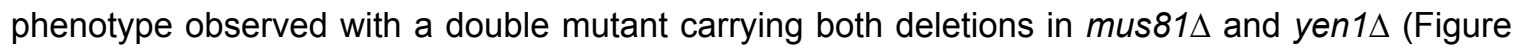
$6 \mathrm{~B}$ and $\mathrm{C}$, Table S10). The decrease in crossover formation was paralleled by an increase in Break-Induced Replication (BIR) events (Figure 6B and C, Table S10). Using an ectopic recombination assay (48) (Figure $6 \mathrm{D}$ and $E$ ), we detected a decrease in viability after the

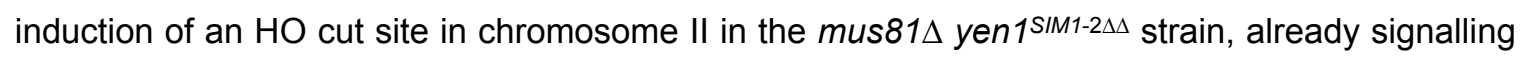
a defective crossover resolution resulting in a number of unviable events (Figure 6F). This survival decrease probably reflects a BIR increase that in this test leads to lethality by the loss of essential genes in the chromosome II distal arm. The number of crossovers quantified by southern blotting analysis of the survivors showed a nearly $50 \%$ reduction in the crossover yields

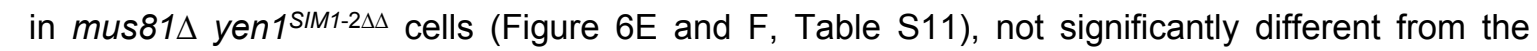
levels detected in mus81 8 yen $1 \Delta$ cells.

\section{$<$ Figure 6>}


319

320

321

322

323

324

325

326

327

328

329

330

331

332

333

\section{Discussion}

In the present work we aimed to understand whether the Yen1 nuclease depends on interactions with Sumoylated partners to be able to act accurately and promptly on its substrates. We have demonstrated that in addition to being Sumoylated, Yen1 is also able to interact non-covalently to Sumoylated chains and Sumo monomers through at least two Sumo-interacting motifs in its Cterminal region (Figure 1). Previous reports suggested covalent Sumoylation occurring in the Cterminal region of Yen1 was responsible for the Smt3-interaction detected in a two-hybrid assay (40), our results are in agreement with the original observation of a C-terminal motif mediating such Smt3-interaction but we conclude this results are compatible with a non-covalent interaction mediated by the two SIMs, leading to a covalent modification of Yen1. We have demonstrated such non-covalent interaction with dedicated retention assays using either immobilized GSTSmt3 or pre-polymerized poly-(6HIS)-Smt3 coupled to a Cobalt HisPur Superflow agarose matrix (Figure 1) in a strategy similar to that used to validate other sumoylated protein's SIMs $(36,49$ 51). Moreover, we further confirmed the nearly complete loss of in vivo Sumo covalent modification in the SIM defective Yen1 using 6His-Smt3 denaturing pull-downs (Figure 3A). Our Yen1 SIM mutant acts thus as an in vivo Sumo-less variant without requiring a large number of Lysine substitutions, which can sometimes result in the protein's destabilization. While direct Yen1 sumoylation depends strongly in the presence of the identified SIM motifs, we conclude those are not required to mediate interaction to the sumoylation machinery per se, as we can observe full sumoylation patterns after an in vitro sumoylation reaction (Figure $3 \mathrm{~B}$ ) and we also detect residual forms of sumoylated Yen1 in our denaturing pull-down assays (Figure 3A) displaying the regular band pattern of in vivo sumoylated Yen1, and not the absence of these forms that is obtained when Siz1 and Siz2 are removed (13). However, we conclude that this direct sumoylation of Yen1 is largely prevented in the cells by a faulty localization via SIMs to specific nuclear sites as suggested by the inability of the SIM mutant to accumulate in foci in subnuclear localizations previously characterized (13) (Figure 4). Accordingly, while the absence of the SIMs in Yen1 has no effect on its catalytic activity (Figure 3C), we have detected a suboptimal function of these mutants in the cells, leading to phenotypes of chromosome missegregation and DNA damage sensitivity similar to those observed for a null mutant in combination with a deletion of the partially redundant cell's major resolvase activity mediated by the heterodimer Mus81-Mms4 (Figures 2, 4 and 5) (5). The impaired localization not only correlates with a sub-optimal function of Yen1 in response to spontaneous damages under normal growth conditions and exogenous treatments with genotoxic agents, but also decreases the number of crossing-over that can be observed after a single DSB induction in two different settings (Figure 6). While inactivation of both SIMs identified in the C-terminal region is necessary 
355 to impair non-covalent binding to Sumo, the mutation we introduced in SIM1 seems to achieve a

356 stronger phenotype alone than the one in SIM2. Nonetheless, we did not perform an optimized

357 serial mutation of each motif and thus we can't exclude that both SIM motifs contribute with equal

358 importance to Sumo binding in the cells.

360 Our results are in line with group modification (35) and would suggest a local enrichment of 361 multiple Sumoylated proteins together with free Smt3 and the Sumoylation machinery when 362 persistent recombination intermediates are revealed during anaphase. While Sumoylation has 363 been previously shown to play important roles in the fine-tuning of DNA repair processes, our 364 study highlights the importance of Sumoylation for genome maintenance processes occurring in anaphase, and probably disconnected to previous Sumoylation cascades influencing HR proteins. Several key proteins acting in anaphase, like Condensin subunits and chromosomal passenger complex (CPC) components, have been described to be Sumoylated $(20,52,53)$. It is thus of great interest to continue studying Yen1 functional interactions in conditions that are greatly transient an ephemeral, and determine which other factors ensure prompt Yen1 recruitment to its activity sites during its anaphase activity window, thus influencing the delicate balance between chromosome segregation and genome integrity.

\section{Materials and Methods}

Yeast Strains and Growth Conditions. S. cerevisiae strains used in this study are derivatives of the W303c background and are listed in Table S1. The Yen1-FX-GFP allele was made by inserting a Factor $X$ site and the GFP epitope from pGAD-Yen1-GFP(54) between amino acids D753 and S754 at the C-terminus of Yen1 using dedicated oligonucleotides and was cloned into TOPO-pYES2 (Invitrogen) to allow controlled expression by Galactose induction, all plasmid derivatives are listed in Table S2. Mutants in the different designated loci where either obtained by crossing or by gene replacement with the indicated selective cassettes. Cells were typically grown in YP (1\% yeast extract; $2 \%$ peptone) or SC media with alternatively $2 \%$ glucose, $2 \%$ raffinose or $2 \%$ galactose in strains under inducible conditions. A modified medium (SC with $0.17 \%$ YNB without ammonium sulfate, $0.1 \%$ proline and $0.003 \%$ SDS) was used for the Smt3 pull-down assays.

Western Blot analyses. If not stated otherwise, proteins were extracted by the TCA 389 (Trichloroacetic acid) method. For routine monitoring, samples were loaded into $7.5 \%$ Tris390 Glycine stain-free pre-casted gels (BioRad). Samples from pull-downs analyses were loaded into 
391

392

393

394

395

396

397

3-8\% gradient NuPAGE Tris-Acetate gels (ThermoFisher). Gels were transferred using a semidry transfer machine (BioRad) to PVDF membranes and hybridized with the appropriate antibodies in 5\% w/v nonfat dry milk, 1X TBST buffer. Antibodies for anti-HA-HRP (3F10, Roche), anti-Smt3 (B. Palancade), anti-Pgk1-HRP (22C5D8, Abcam) were used at the suggested dilutions and revealed using an ECL reagent (Advansta). When required, HRP-conjugated secondary antibodies from Cell Signaling were used at 1/10000 dilution.

Microscopy and Cell Biology Methods. Live cell imaging was performed with a Spinning Disk Confocal Microscope (CSU-W1, Yokogawa), with an electron multiplying charge device camera (ANDOR Zyla sCMOS) and a $\times 60 / 1.35$ numerical aperture objective at $30^{\circ} \mathrm{C}$. Cells were centrifuged and plated as a droplet between an SC agarose pad and a glass slice (55). Images were recorded with $17 \mathrm{z}$-sections with $0.5 \mu \mathrm{m}$ spacing for each wavelength at a time. Video recordings were built with images taken every 2 minutes. Metamorph was used for image acquisition, and analysis was performed using Image J-Fiji (56).

For Yen1 foci observations, cells were grown in SC medium without uracil (SC-URA 2\% raffinose), GFP-Yen1 was induced in a short burst of 30 min with Galactose at $2 \%$, followed by addition of Glucose at $2 \%$. For acute exposure to DNA damage, cells were treated with MMS $0.01 \%$ for $15 \mathrm{~min}$ at room temperature and were washed once with fresh SC-URA $2 \%$ glucose before continuing the experiment. Aliquots were taken at the indicated times. Cells showing an accumulation of spots were measured at maximum projection of the GFP channel. Statistical significance was determined by the $x 2$ test using contingency tables with the number of cells observed in each different category.

For segregation monitoring using strains with the lacO/GFP-Lacl array, all cells were recorded for a duration of $2 \mathrm{~h}$ minimum in their agarose pads. Individual cells were identified with an ongoing chromosome segregation. To determine segregation duration a start point was determined as the signature S-phase bud was the smallest yet discernable. At this point, only one foci of GFPtagged chromosome fluorescent markers is visible. The cell is followed until the dot separates in two and resides durably in the daughter cells. The ending time point is taken at the last frame of definitive separation of the fluorescent foci. The duration of the movement of the two separate dots was reported for each individual cell under monitoring, cells with dots moving together for the whole duration of the time-lapse were assigned as non-disjunction and their segregation time was not used to establish the average segregation time.

Sumoylation assays and Smt3-bound retention assay. In ex vivo Sumoylation assays, the wild-type or mutant Yen1-HA was produced from a pYES2 vector and immuno-precipitated from 
426 cell lysates as described (13). Eluates were subjected to Sumo conjugation and ligation as

427 described (57).

428 For Smt3-retention assays, 6x-His-Smt3 was purified from BL21 E.coli cells using a Ni-NTA 429 affinity column (Qiagen) following manufacturer indications. Smt3 protein was eluted with $250 \mathrm{mM}$ 430 imidazole before being dialyzed using a G2 Slide-a-Lyzer cassette (Thermo Fisher) with a $10 \mathrm{kDa}$ 431 cut-off. Purified Smt3 was subjected to a self-conjugation reaction by adding Aos1-Uba2 and 432 Ubc9 and ATP as described (57) and the reaction was subjected to a second purification in a 433 Cobalt HisPur Superflow agarose matrix (Thermo Fisher) to generate poly-Smt3 retention 434 column. Equal amounts of Yen1 or its mutant were added to the non eluted matrix containing 435 poly-Smt3 bound in $\mathrm{E}$ buffer $\left(20 \mathrm{mM} \mathrm{NaH} \mathrm{PO}_{4}, 300 \mathrm{mM} \mathrm{NaCl}, 5 \mathrm{mM}\right.$ Imidazole, $\mathrm{pH}$ 7.4) and 436 binding was allowed for $60 \mathrm{~min}$ at $4^{\circ} \mathrm{C}$. Mini-Columns were then centrifuged to remove the buffer 437 and non-retained proteins, washed 5 times in washing buffer (E buffer $12.5 \mathrm{mM}$ Imidazole) and 438 eluted in denaturing conditions with Laemmli buffer at $95^{\circ} \mathrm{C}$. The eluates were loaded into $4-15 \%$ 439 SDS-PAGE gradient gels and immunoblotted. GST-Smt3 retention assays were performed as 440 described (36) with purified GST-Smt3 obtained by expression of pGEX-4T1-Smt3 into BL21

441 E.coli cells. Briefly, purified GST-Smt3 was incubated with glutathione matrix during 40 min at $4^{\circ} \mathrm{C}$ 442 in GST buffer (25 mM Tris-HCl (pH8.0), 150mM NaCl, 1mM DTT). Then matrix was washed 443 before to load purified wild-type or mutated Yen1. Mixtures were incubated $3 \mathrm{~min}$ at $4^{\circ} \mathrm{C}$ before to 444 wash the glutathione matrix and elute retained proteins for HA and anti-GST immuno-bolt 445 analysis.

Cycloheximide chase experiments. Cycloheximide chase experiments were essentially done 448 as reported (13). Cultures grown in SC complete modified media $(0.1 \%$ proline $0.017 \%$ YNB 449 without ammonium sulfate, $0.0003 \%$ SDS) were diluted to $\mathrm{OD}_{600}=0.2$ and synchronized with 450 alpha factor $(3 \mu \mathrm{M})$ for $2 \mathrm{~h}$. Once synchronized, cells were treated with cycloheximide $(250 \mu \mathrm{g} / \mathrm{ml})$ 451 in fresh media, to inhibit proteins new synthesis, and released from the G1 arrest. Samples were 452 taken at indicated time points and analyzed by TCA extraction and western blotting.

454 Denaturing Histidine pull-downs. For 6xHIS-Smt3 pull-downs, strains containing the 455 expression vectors or the control empty plasmid were grown in SC-LEU modified medium (0.1\% 456 proline, $0.017 \%$ YNB without ammonium sulfate). Cells were allowed to grow to $\mathrm{OD}_{600}=0.3$ when $457 \mathrm{CuSO}_{4}$ was added at $100 \mu \mathrm{M}$ final concentration in a volume of $100 \mathrm{ml}$. After $1 \mathrm{~h}$, MMS was 458 added to $0.3 \%$ and cells were collected $3 \mathrm{~h}$ later. Cells where lysed under denaturing conditions 459 and Sumo-conjugated proteins where isolated and analyzed by western blot using a Nu-PAGE 460 Tris-acetate 3-8\% gradient gel, basically as previously described (13). In dna2-2 strains (LEU2), a 
plasmid expressing under Galactose induction Flag-6His-Smt3 (URA3) was used instead of the

462 Cu-inducible.

Synthetic DNA substrates and Yen1 resolvase activity assays. The synthetic HJ-XO was prepared by annealing the Cy5-X0-1, X0-2, X0-3 and X0-4 oligonucleotides (Sigma-Aldrich) in a buffer containing $50 \mathrm{mM}$ Tris- $\mathrm{HCl}(\mathrm{pH} 7.5), 10 \mathrm{mM} \mathrm{MgCl}, 100 \mathrm{mM} \mathrm{NaCl}$ as described (46). The annealing product was analyzed in a native PAGE to verify the presence of a HJ structure. To test Yen1 activity, an enzymatic reaction was performed in $10 \mu \mathrm{l}$ cleavage buffer $(50 \mathrm{mM}$ Tris- $\mathrm{HCl}$ (pH 7.5), $1 \mathrm{mM} \mathrm{MgCl}_{2}, 1 \mathrm{mM} \mathrm{DTT}$ ) containing $25 \mathrm{nM}$ of Cy5-labled HJ XO substrate, and equal amounts of immunoprecipitated Yen1 or its SIM-defective mutant. After incubation at $30^{\circ} \mathrm{C}$ for 1 $\mathrm{h}$, the reaction was stopped by adding $2.5 \mu \mathrm{l}$ of stop buffer (100 mM Tris-HCl (7.5), $50 \mathrm{mM}$ EDTA, $2.5 \% \mathrm{SDS}, 10 \mathrm{mg} / \mathrm{ml}$ proteinase $\mathrm{K}$ ) and further incubated for $30 \mathrm{~min}$ at $37^{\circ} \mathrm{C}$. Cleavage products were migrated in 10\% native PAGE, scanned using a Typhoon FLA 9500 Biomolecular Imager and the images were analyzed with ImageQuant (GE Healthcare).

DSB-induced recombination assays. The diploid recombination assays were performed as described previously (for a detailed protocol see (47)). The reporter diploid strains that contain 2 ade2 hetero-alleles were cleaved by induction of I-Scel in its ade2-I allele and allowed to repair with its ade2-n allele under non-selective conditions to give rise to either ADE2 or ade2-n repair products in three types of colonies (red, white and sectored). Each recombinant colony was scored with its two recombination events (for each repaired sister chromatid) and considering the possible segregation patterns in daughter cells. Frequencies of the recombination events were normalized to the galactose vs glucose plating efficiency. The distribution of $\mathrm{CO}$ and $\mathrm{NCO}$ in the ectopic recombination assay based in chromosomes V and II (48) were addressed by southern blot hybridization of ApaLI-Pvull digested genomic DNA from cell populations growing in YPRaffinose after galactose induction of $\mathrm{HO}$. Membranes were hybridized with a URA3 radiolabeled probe and results were normalized relative to the galactose versus glucose plating efficiency of the strains as described (3).

\section{Acknowledgments}


497 the Sumoylation assays. We thank the members of the UMR9019 CNRS for the useful 498 discussions and suggestions. We thank Fondation ARC pour la Recherche sur le Cancer and 499 donators from Natixis for their support of our research. GM is a full-time INSERM researcher at 500 the CNRS. IT is a full-time CNRS researcher. HD has benefited from a doctoral fellowship from 501 the French Education Ministry.

502

\section{Funding}

504

505 This study was supported by grants to GM from the Gustave Roussy Foundation (FGR-JC6, 506 funded in part from donations from Natixis) and from Fondation ARC pour la Recherche sur le 507 Cancer (PJA 20181208087).

508

509 Data Availability

510 The data that support the findings of this study are available from the corresponding author upon 511 reasonable request 


\section{Figure Legends}

515 Figure 1. Yen1 contains two Sumo Interacting Motifs (SIMs) in its C-terminal domain

516 (A) Diagram showing the conserved domains of Yen1 and the positions of the regulatory Cdk1517 phosphorylation sites. Amino acid 354 shows the cut-off point for truncated forms of Yen1 in Two518 Hybrid assays. The two identified candidate SIMs are shown near the Nuclear localization 519 sequence (NLS). (B) A Two-hybrid assay was performed with strains carrying the indicated 520 Activator Domain (AD) and DNA Binding Domain (BD) fusions to test interaction between Yen1 521 and Smt3 (SUMO), and the Yen1 critical domains for such interaction. Mutations D635A D636A 522 D637A for SIM1 $\Delta$ and V675A E677A for SIM2 $\Delta$ were introduced to test for the putative SIMs. 523 SIM1 $-2 \Delta \Delta$ is used for the combined mutations. Strains were grown on selective media lacking 524 Leucine and Tryptophan and spotted in selective media also lacking Histidine to reveal interaction 525 of the proteins fused to the $A D$ and $B D$ domains. Non-specific interactions were minimized by the 526 addition of 3-aminotriazole (AT). (C) Purified GST-Smt3 was bound to a Glutathione resin and 527 either purified wild-type or the SIM mutant Yen1 proteins were then loaded to the resin. The 528 retained fractions of Yen1 were eluted after several washes and detected by immuno-blotting. (D) 529 SUMO-retention assay using in vitro generated poly-Smt3 immobilized into a Cobalt HisPur 530 Superflow agarose matrix. Purified Yen1 was added to the pre-bound Smt3 and after a binding531 time and washing, columns were eluted in denaturing conditions and the eluates inspected by 532 western blot for the presence of Yen1 (anti-HA, left panel) and the pre-bound Smt3 chains (right 533 panel). (E) Immuno-blotting of the inputs and eluates of the retention assay (as in D) comparing 534 the ability of wild-type or SIM-mutant Yen1 to bind poly-Smt3.

536 Figure 2. Mutation in Yen1 SIMs has no impact to its CDK1 regulation and nuclear shuttling but sensitizes cells to DNA damage

538 (A) Cells carrying an endogenous histone Hta2-mCherry marker and chromosomally - HA tagged 539 versions of Yen1 wild-type and the different SIM mutants were transformed with a plasmid 540 carrying an equivalent version of Yen1 fused with GFP at its C-terminal region. Cells were grown 541 on selective media and observed using a spinning-disk microscope after a brief induction with 542 galactose. Shuttling of the protein from cytoplasm to the nucleus can be observed in 543 representative fields displaying cells with nuclear excluded Yen1 (S-phase and early G2-M) and 544 nuclear localized Yen1 (anaphase to G1) for the indicated GFP-tagged proteins. (B) 545 Quantification of the relative amount of GFP signal detected into the nucleus over the overall 
546 signal for the indicated strains in cells classified by their cell-cycle status (C) Strains with a 547 chromosomally inserted copy of -HA tagged wild-type Yen1 or its double SIM mutant (Yen1SIM1-

$5482^{2 \Delta}$ ) were synchronized with alpha factor and released into fresh medium to monitor the 549 modification of the protein through the cell cycle by immunoblot (left). Both unmodified and 550 phosphorylated Yen1 are indicated. Average levels of endogenous Yen1 were normalized with 551 PGK1 protein in triplicate experiments (right). (D) Sensitivity to different DNA damaging agents 552 and drugs was determined by spotting serial dilutions of strains carrying different Yen1 mutants in 553 its SIM in a MUS81 deleted background for the indicated media. (E) Survival curves to the agents 554 tested in (C) were established by counting colony forming units of the different strains after plating 555 in YPD containing the indicated doses of drugs in replicate trials. Survival was normalized per trial 556 with its respective control YPD counts and the average \% survival is plotted in the graphs (+/557 SEM). Significance was estimated by the student T-test at $P<0.05\left(^{*}\right)$ and $P<0.01\left(^{* *}\right)$, see Table 558 S3. Additional data related to this figure is found in Figure S2.

Figure 3. Mutation in Yen1's SIMs does not alter its activity or its sumoylation in vitro but prevents sumoylation in vivo.

562 (A) Strains carrying endogenous copies of $-\mathrm{HA}$ tagged wild type Yen1, Yen1 ${ }^{\mathrm{SIM} 1 \Delta}$, Yen1 ${ }^{\mathrm{SIM} 2 \Delta}$ and 563 Yen1 $1^{\text {SIM1-2 }}$ mutants, with (+) or without (-) the plasmid pCUP-6xHIS-Smt3, were grown in the 564 presence of MMS $0.3 \%$. Cells were lysed and lysates subjected to a denaturing Ni-NTA pull-down 565 followed by immunoblot analysis. Yen1 was detected by anti-HA. Membranes were subsequently 566 probed with anti-Smt3. Prior to Ni-NTA pull-down, input samples were taken from the lysates and 567 were analysed by immunoblotting for the levels of Smt3 induction (Anti-Smt3) and relative protein 568 amounts (Anti-PGK1, Anti-HA) of each lysate (input panels). (B) Purified Yen1-HA and Yen1 SIM569 mutant variants were subjected to an in vitro sumoylation reaction containing Siz2, Aos1-Uba2, 570 Ubc9 and Smt3-3KR and ATP and subjected to Tris-Acetate PAGE for comparison of their 571 sumoylation patterns after immunoblotting with anti-HA. The reaction without the ligase Siz2 is 572 shown at the right of each panel. Quantification of the relative \% of sumoylation forms (R.I.) and 573 unmodified forms is shown at the bottom and more in detail in Figure S4 (C) Activity of Immuno574 precipitated Yen1 was tested in a cleavage reaction using synthetic Holliday Junctions (HJ) made 575 with oligonucleotides and labeled with Cy5. The DNA products were run in non-denaturing PAGE 576 and revealed by the fluorescence of the Cy5 labeled oligonucleotide. 


\section{Figure 4. Mutation in the SIMs of Yen1 prevents foci accumulation in G2/M.}

580 (A) Cells with an endogenous copy of Hta2-mCherry and YEN1-HA expressing Yen1-GFP from 581 an inducible vector were observed under a spinning-disk microscope after a brief induction with 582 galactose. The white triangles denote the presence of Yen1-GFP foci. (B) Chromosomally tagged 583 Yen1-HA wild-type and SIM mutant in the indicated genetic backgrounds and carrying its 584 corresponding Yen1-GFP expressing plasmid (blue graphs for WT copy, red for SIM mutant) 585 were observed under the microscope after a brief induction. Cells from the indicated conditions 586 were classified according to its cell cycle phase and the presence or absence of Yen1 foci. Violin 587 Plots display the distribution of G2/M cells showing no foci, 1-2 foci or more than 2 foci for each 588 strain. Counting was performed for over 400 distinct G2/M cells for each strain over several 589 independent trials. Asterisks represent statistical significance in a $x 2$ test $p<0.0001{ }^{* * *}$ ), see 590 tables S4, S5, S6 and S7 for details.

592 Figure 5. Mutation in the SIMs of Yen1 prevents foci accumulation in s/x8 $\Delta$ cells during 593 G2/M and impacts chromosome segregation in a mus81 $\Delta$ background.

594 (A) Cells containing a deletion on SLX8 were observed for their distribution of foci of the different 595 variants of Yen1-GFP. Violin plots display the distribution of cells and asterisks denote statistical 596 significance in a Chi-square test at $\left.\mathrm{P}<0.0001{ }^{* * *}\right)$, see table S8. (B) Cells from the indicated 597 genotypes were arrested in $\mathrm{G} 1$ and released in the presence of cycloheximide with samples 598 being taken at the indicated time points. Total protein extracts were inspected by immunoblot for 599 the presence of Yen1-HA and their intensity quantified relative to the loading control obtained by 600 stain-free imaging of the gels (BioRad). Relative amounts of Yen1 are plotted in the graph to 601 facilitate comparison (+/- SD). (C) Diagram showing chromosome segregation in cells harboring 602 a lacO/GFP-Lacl array tag on chromosome VII. To discriminate cells with timely chromosome 603 segregation from those presenting aberrant segregation (delayed segregation or non-disjunction) 604 a $2 \mathrm{~h}$ limit of observation was implemented. Two sets of representative actual images of a normal 605 segregation pattern and a non-disjunction pattern are shown below the diagram. (D) Over 400 606 cells per strain were individually counted and are represented in violin plots according to the time 607 spent to segregate the lacO/lacl array. The median segregation time is indicated excluding cells 608 with non-disjunctions. Statistical relevance of the differences observed between the number of 609 non-disjunctions of the different strains was determined by the Chi-square test at $P<0.0001$, see 610 table S9. 
612 Figure 6. Crossover formation is impaired in cells containing the mutant version of Yen1 613 inactivating both SIMs in a mus81 $\Delta$ background.

614 (A) Diagram showing the chromosome XV based DSB-induced recombination reporter. (B) 615 Recombination outcomes in red-white (ade2/ADE2) sectored colonies of the indicated strains, 616 normalized to their Plating Efficiency (PE) in Galactose compared to Glucose. (C) Recombination 617 outcomes combining the results obtained for all types of colonies (full red, full white and sectored) 618 of the indicated strains, normalized to their Plating Efficiency (PE) in Galactose compared to 619 Glucose. Statistical significance for B and C was determined by the Chi-square test at $P<0.05$, 620 see table S10. (D) Diagram showing the chromosome II-V based ectopic DSB-induced 621 recombination reporter and its expected outcomes during physical analysis. (E) Representative 622 southern blot analysis of the indicated strains after genomic DNA digestion with the restriction 623 enzymes as highlighted in diagram $D$ and hybridization with a radiolabeled probe against the 624 URA3 locus. (F) Quantification of at least three independent southern blot analyses is plotted 625 relative to PE (Galactose vs Glucose). Statistical significance was determined by the Student T626 test at $P<0.05$, see table S11. 

structures of crossover and noncrossover intermediates during gap repair in yeast: implications for recombination. Mol Cell 38(2):211-222. mitotic recombination in Saccharomyces cerevisiae. Genetics 198(3):795-835. Mazon G \& Symington LS (2013) Mph1 and Mus81-Mms4 prevent aberrant processing of mitotic recombination intermediates. Mol Cell 52(1):63-74. anaphase bridge formed by noncanonical Holliday junctions. Nat Commun 5:5652. reciprocal exchange during mitotic recombination to maintain genome integrity in budding yeast. Mol Cell 40(6):988-1000. of the resolution of DNA recombination intermediates during meiosis and mitosis. Cell 147(1):158-172.

Gallo-Fernandez M, Saugar I, Ortiz-Bazan MA, Vazquez MV, \& Tercero JA (2012) Cell cycle-dependent regulation of the nuclease activity of Mus81Eme1/Mms4. Nucleic Acids Res 40(17):8325-8335. induces aberrant replication and deleterious crossover. EMBO J 32(8):11551167. the resolution of DNA repair intermediates linked to stalled replication. Genes Dev 28(14):1604-1619. Bastin-Shanower SA, Fricke WM, Mullen JR, \& Brill SJ (2003) The mechanism of Mus81-Mms4 cleavage site selection distinguishes it from the homologous endonuclease Rad1-Rad10. Mol Cell Biol 23(10):3487-3496.

Blanco MG, Matos J, \& West SC (2014) Dual control of Yen1 nuclease activity and cellular localization by $\mathrm{Cdk}$ and $\mathrm{Cdc} 14$ prevents genome instability. Mol Cell 54(1):94-106.

Eissler CL, et al. (2014) The Cdk/cDc14 module controls activation of the Yen1 holliday junction resolvase to promote genome stability. Mol Cell 54(1):80-93. 


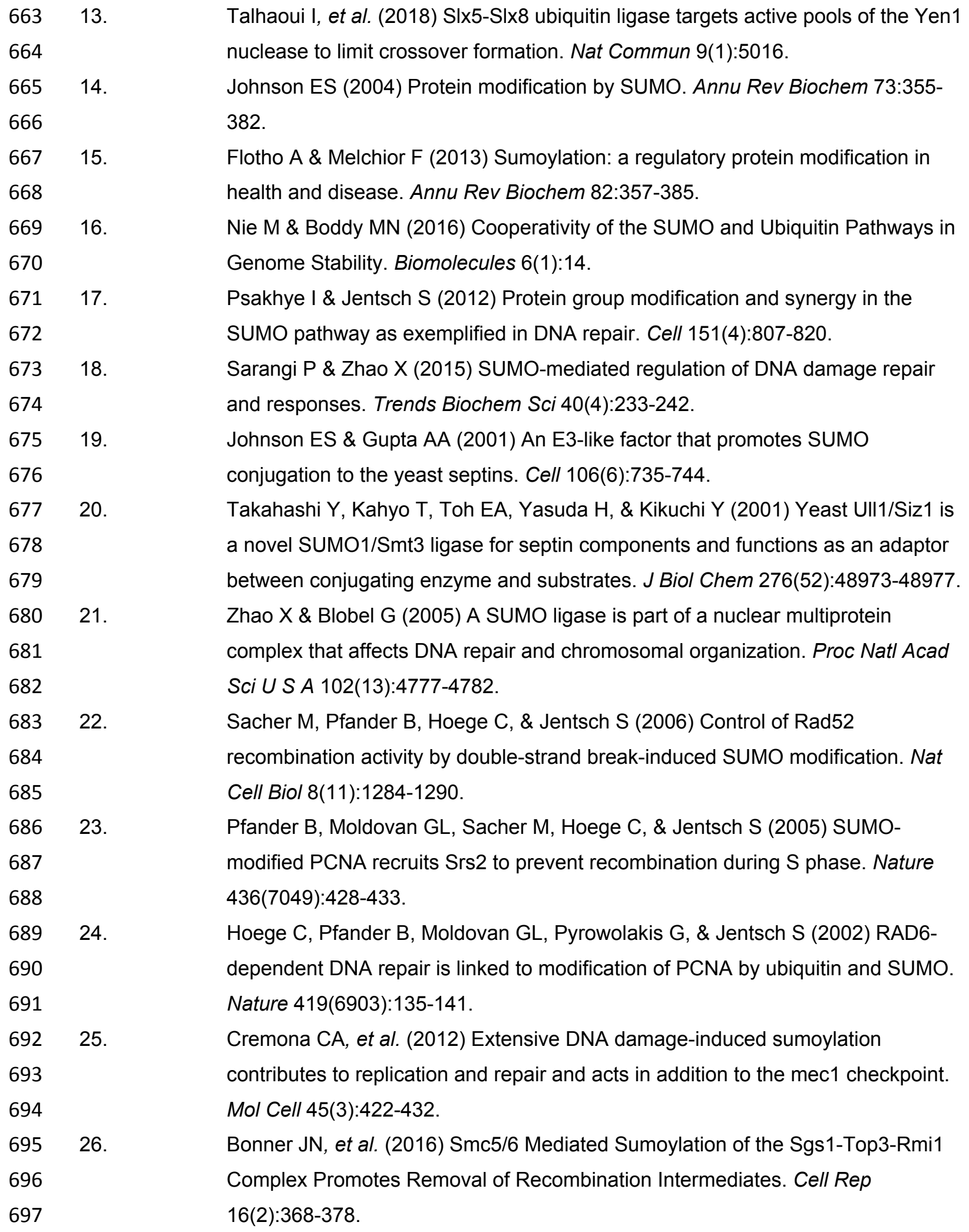

Talhaoui I, et al. (2018) SIx5-SIx8 ubiquitin ligase targets active pools of the Yen1 nuclease to limit crossover formation. Nat Commun 9(1):5016. Johnson ES (2004) Protein modification by SUMO. Annu Rev Biochem 73:355382.

Flotho A \& Melchior F (2013) Sumoylation: a regulatory protein modification in health and disease. Annu Rev Biochem 82:357-385.

Nie M \& Boddy MN (2016) Cooperativity of the SUMO and Ubiquitin Pathways in Genome Stability. Biomolecules 6(1):14.

Psakhye I \& Jentsch S (2012) Protein group modification and synergy in the SUMO pathway as exemplified in DNA repair. Cell 151(4):807-820.

Sarangi P \& Zhao X (2015) SUMO-mediated regulation of DNA damage repair and responses. Trends Biochem Sci 40(4):233-242.

Johnson ES \& Gupta AA (2001) An E3-like factor that promotes SUMO conjugation to the yeast septins. Cell 106(6):735-744.

Takahashi Y, Kahyo T, Toh EA, Yasuda H, \& Kikuchi Y (2001) Yeast UII1/Siz1 is a novel SUMO1/Smt3 ligase for septin components and functions as an adaptor between conjugating enzyme and substrates. J Biol Chem 276(52):48973-48977. Zhao X \& Blobel G (2005) A SUMO ligase is part of a nuclear multiprotein complex that affects DNA repair and chromosomal organization. Proc Natl Acad Sci U S A 102(13):4777-4782.

22. Sacher M, Pfander B, Hoege C, \& Jentsch S (2006) Control of Rad52 recombination activity by double-strand break-induced SUMO modification. Nat Cell Biol 8(11):1284-1290.

23. Pfander B, Moldovan GL, Sacher M, Hoege C, \& Jentsch S (2005) SUMOmodified PCNA recruits Srs2 to prevent recombination during $S$ phase. Nature 436(7049):428-433.

Hoege C, Pfander B, Moldovan GL, Pyrowolakis G, \& Jentsch S (2002) RAD6dependent DNA repair is linked to modification of PCNA by ubiquitin and SUMO. Nature 419(6903):135-141.

25. Cremona CA, et al. (2012) Extensive DNA damage-induced sumoylation contributes to replication and repair and acts in addition to the mec1 checkpoint. Mol Cell 45(3):422-432.

Bonner JN, et al. (2016) Smc5/6 Mediated Sumoylation of the Sgs1-Top3-Rmi1 Complex Promotes Removal of Recombination Intermediates. Cell Rep 16(2):368-378. 
734

735

736

737

738

739

740

741

742

743

744

745

746

747

748

749

750

751

752

753

754

755

756

757

758

759

760

761

762

763

764

765

766

767
40.

41.

42.

43.

44.

45.

46.

47.

48.

49.

50.

51.

52.

53.
Bauer SL, Chen J, \& Astrom SU (2019) Helicase/SUMO-targeted ubiquitin ligase Uls1 interacts with the Holliday junction resolvase Yen1. PLoS One 14(3):e0214102.

Budd ME, et al. (2005) A network of multi-tasking proteins at the DNA replication fork preserves genome stability. PLoS Genet 1(6):e61.

Olmezer G, et al. (2016) Replication intermediates that escape Dna2 activity are processed by Holliday junction resolvase Yen1. Nat Commun 7:13157.

Falquet B, et al. (2020) Disease-associated DNA2 nuclease-helicase protects cells from lethal chromosome under-replication. Nucleic Acids Res 48(13):72657278.

Budd ME, Reis CC, Smith S, Myung K, \& Campbell JL (2006) Evidence suggesting that Pif1 helicase functions in DNA replication with the Dna2 helicase/nuclease and DNA polymerase delta. Mol Cell Biol 26(7):2490-2500. Ip SC, et al. (2008) Identification of Holliday junction resolvases from humans and yeast. Nature 456(7220):357-361.

Benson FE \& West SC (1994) Substrate specificity of the Escherichia coli RuvC protein. Resolution of three- and four-stranded recombination intermediates. $J$ Biol Chem 269(7):5195-5201.

Mazon G, Lam AF, Ho CK, Kupiec M, \& Symington LS (2012) The Rad1-Rad10 nuclease promotes chromosome translocations between dispersed repeats. Nat Struct Mol Biol 19(9):964-971.

Agmon N, Yovel M, Harari Y, Liefshitz B, \& Kupiec M (2011) The role of Holliday junction resolvases in the repair of spontaneous and induced DNA damage.

Nucleic Acids Res 39(16):7009-7019.

Hecker CM, Rabiller M, Haglund K, Bayer P, \& Dikic I (2006) Specification of SUMO1- and SUMO2-interacting motifs. J Biol Chem 281(23):16117-16127.

Xie Y, et al. (2007) The yeast Hex3.SIx8 heterodimer is a ubiquitin ligase stimulated by substrate sumoylation. J Biol Chem 282(47):34176-34184.

Balakirev MY, et al. (2015) Wss1 metalloprotease partners with Cdc48/Doa1 in processing genotoxic SUMO conjugates. Elife 4.

D'Amours D, Stegmeier F, \& Amon A (2004) Cdc14 and condensin control the dissolution of cohesin-independent chromosome linkages at repeated DNA. Cell 117(4):455-469.

Dasso M (2008) Emerging roles of the SUMO pathway in mitosis. Cell Div 3:5. 
bioRxiv preprint doi: https://doi.org/10.1101/2021.10.07.463465 this version posted October 7, 2021. The copyright holder for this preprint (which was not certified by peer review) is the author/funder, who has granted bioRxiv a license to display the preprint in perpetuity. It is made available under aCC-BY 4.0 International license.

76854

769

770

77155

772

77356.

774

$775 \quad 57$

776

777
Kosugi S, Hasebe M, Tomita M, \& Yanagawa H (2009) Systematic identification of cell cycle-dependent yeast nucleocytoplasmic shuttling proteins by prediction of composite motifs. Proc Natl Acad Sci U S A 106(25):10171-10176.

Tran PT, Paoletti A, \& Chang F (2004) Imaging green fluorescent protein fusions in living fission yeast cells. Methods 33(3):220-225.

Schindelin J, et al. (2012) Fiji: an open-source platform for biological-image analysis. Nat Methods 9(7):676-682.

Bretes $\mathrm{H}$, et al. (2014) Sumoylation of the THO complex regulates the biogenesis of a subset of mRNPs. Nucleic Acids Res 42(8):5043-5058. 
A

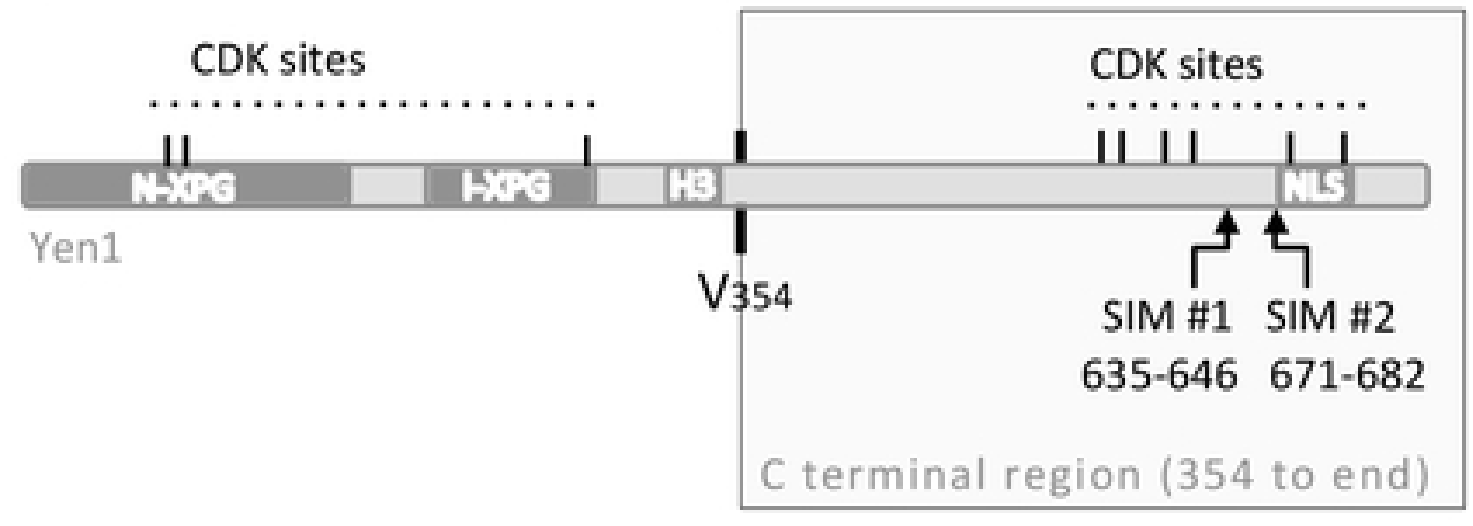

C

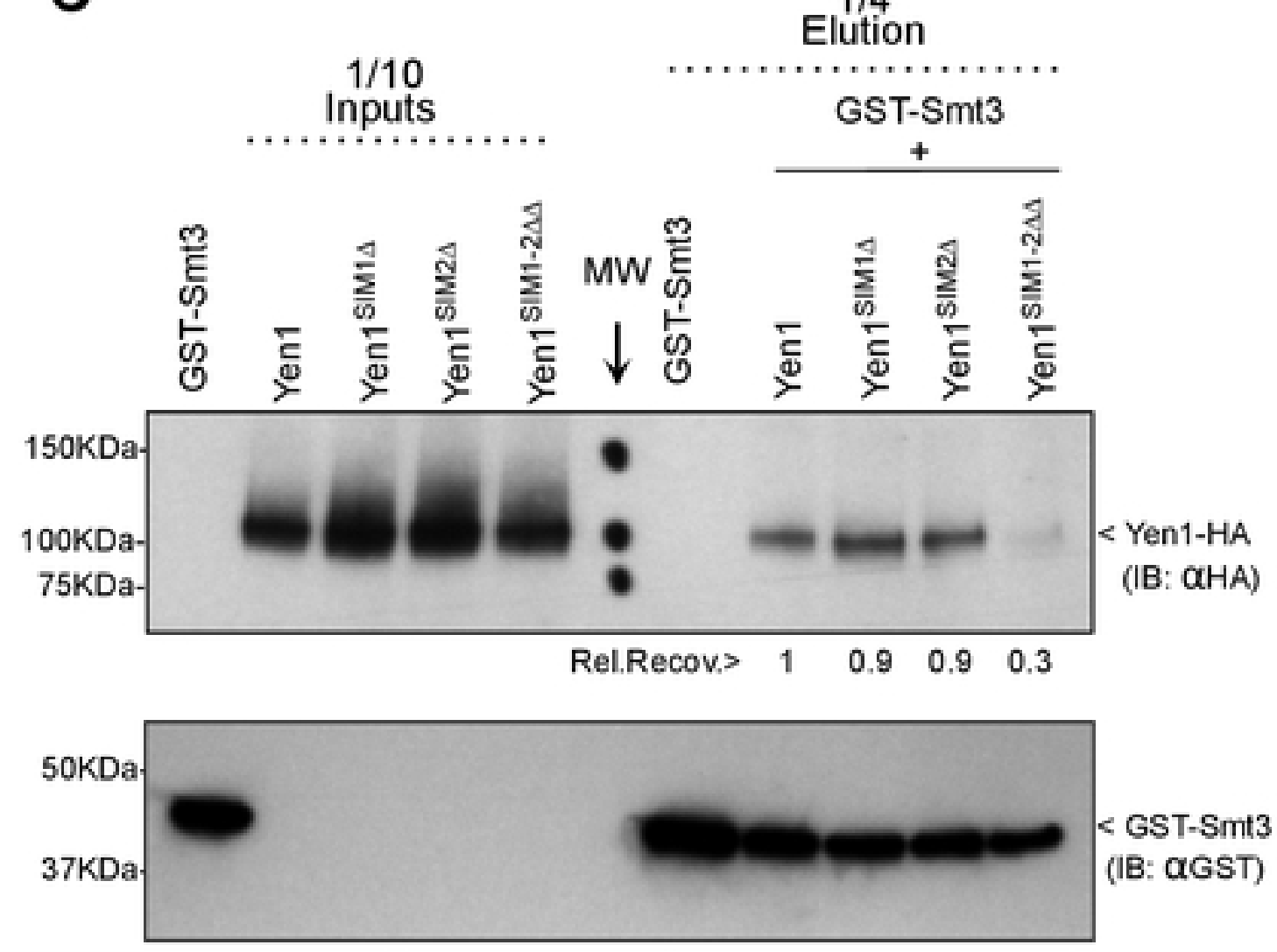

B

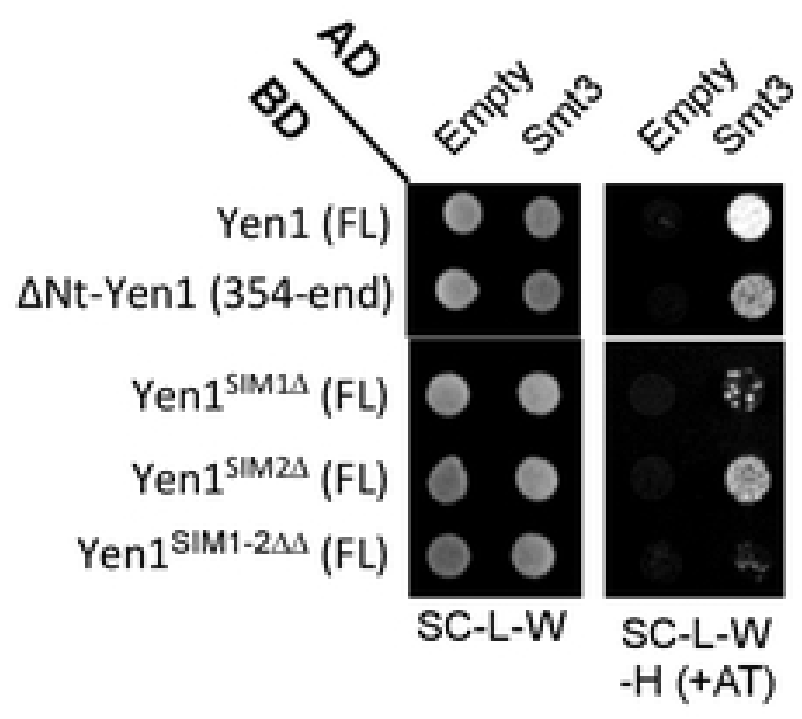

D
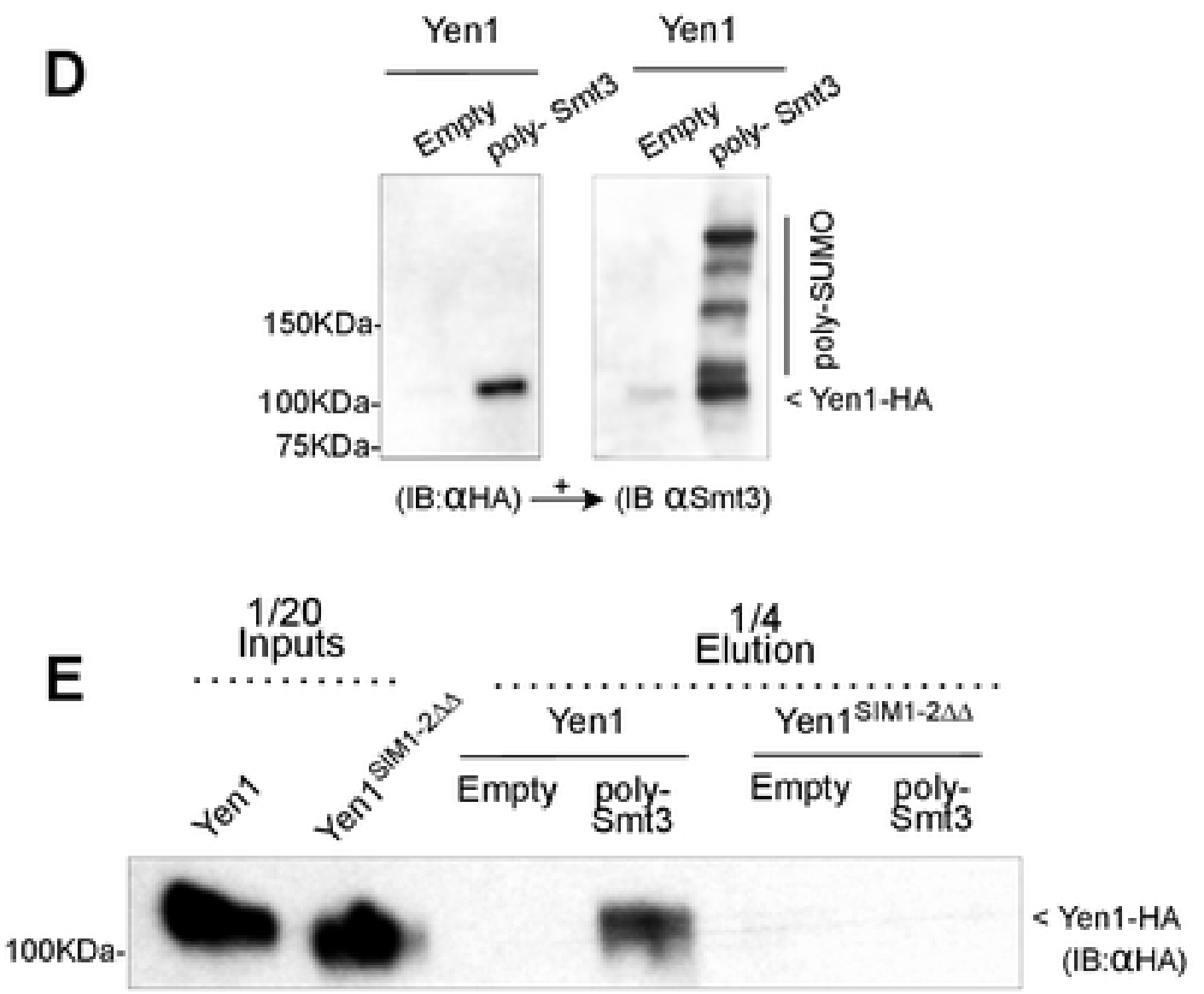

\section{Figure 1}




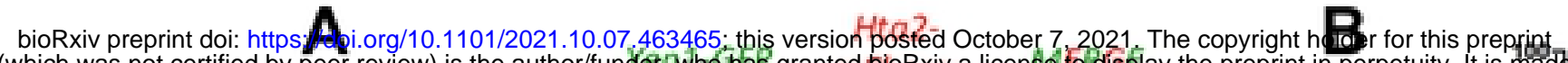
(which was not certified by peer review) is the author/funder('who has granted(bioRxiva license to display the preprint in perpetuity. It is made
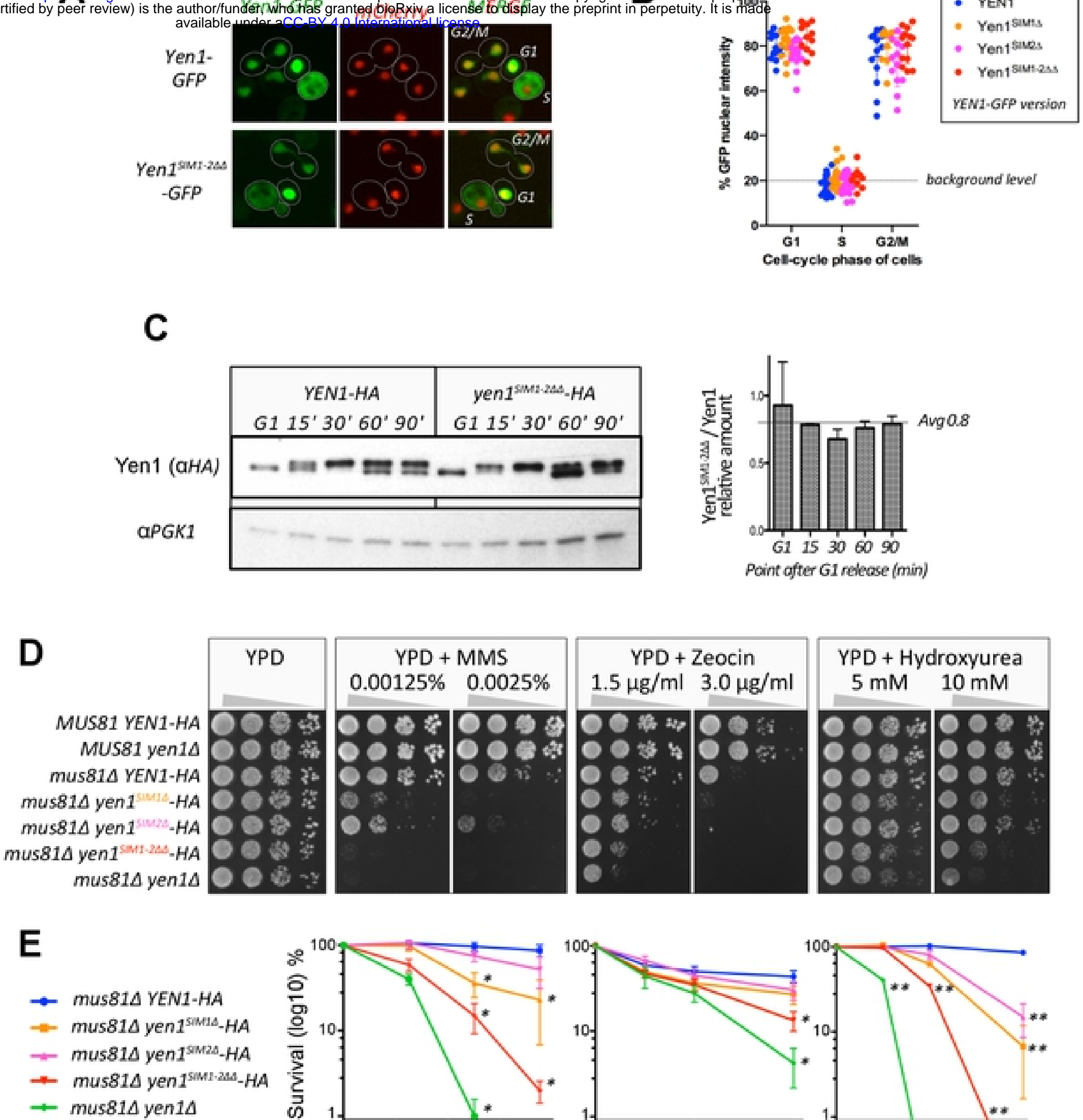

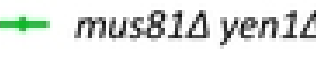
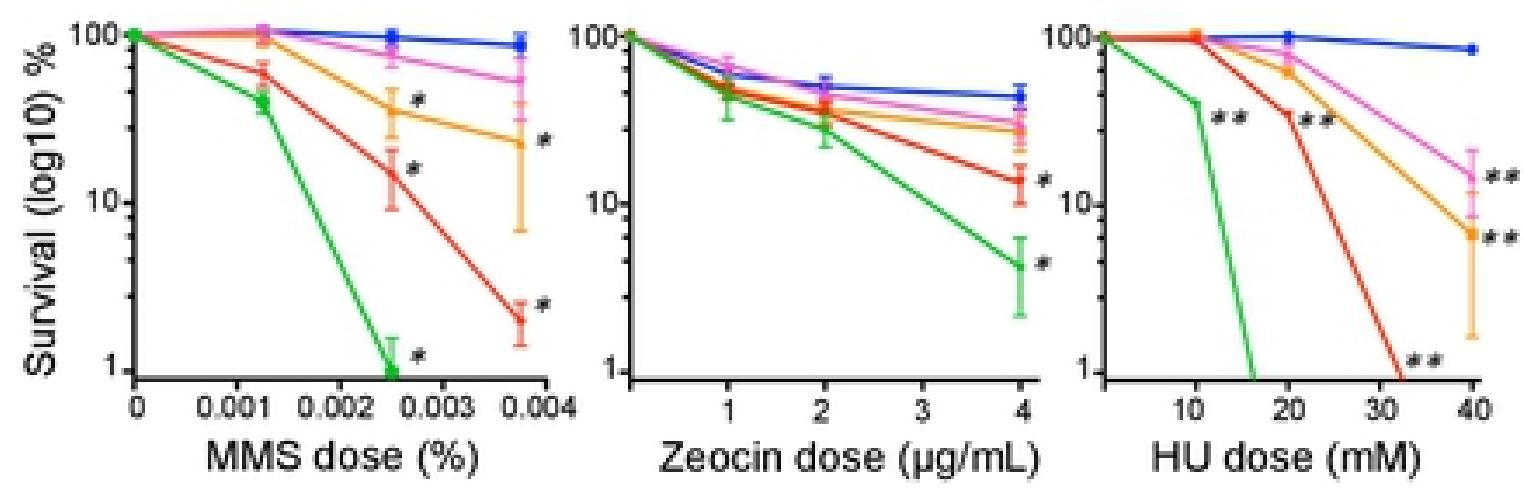

\section{Figure 2}




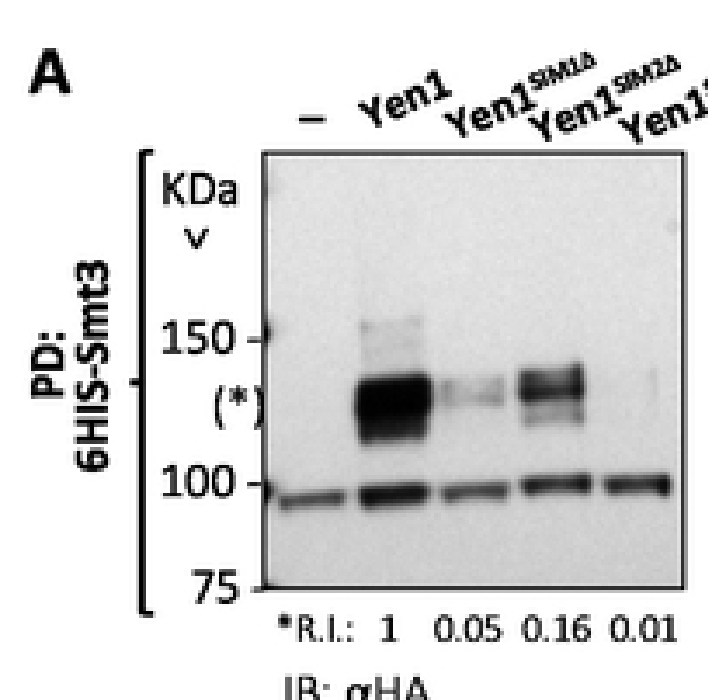

IB: $\alpha \mathrm{HA}$

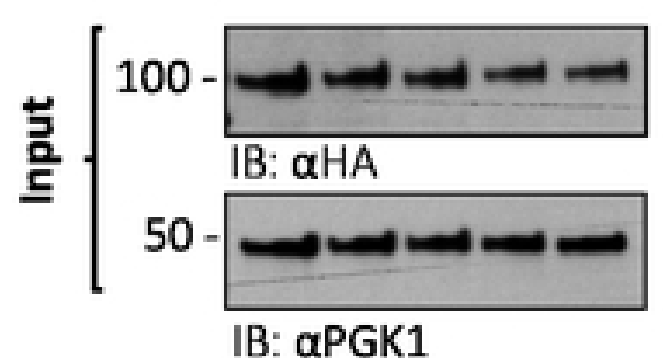

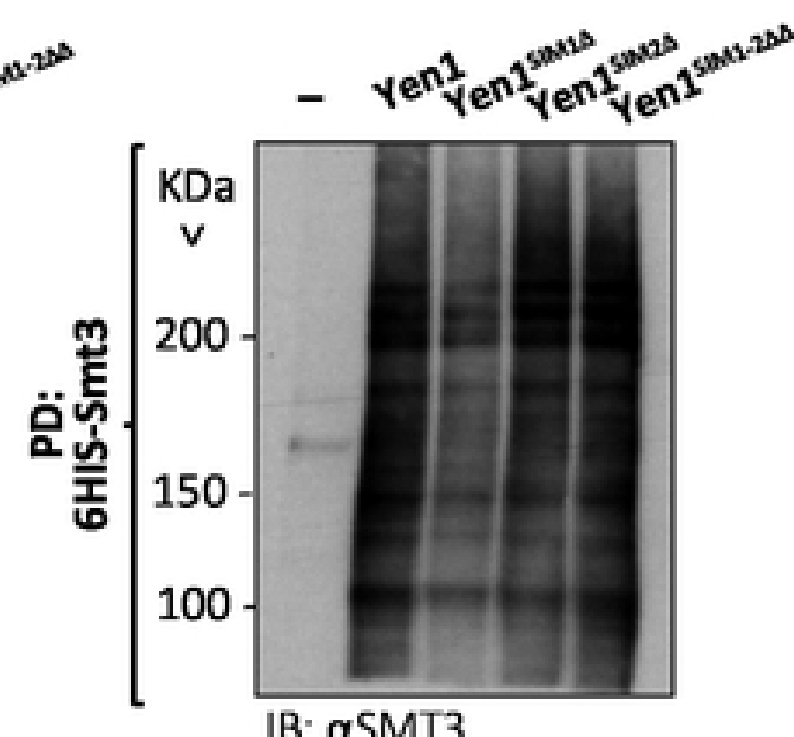

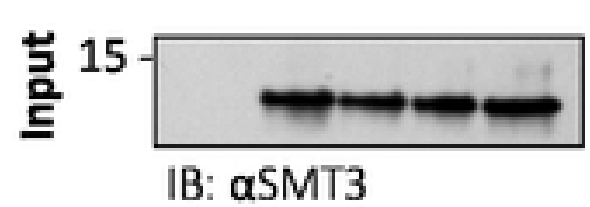

C

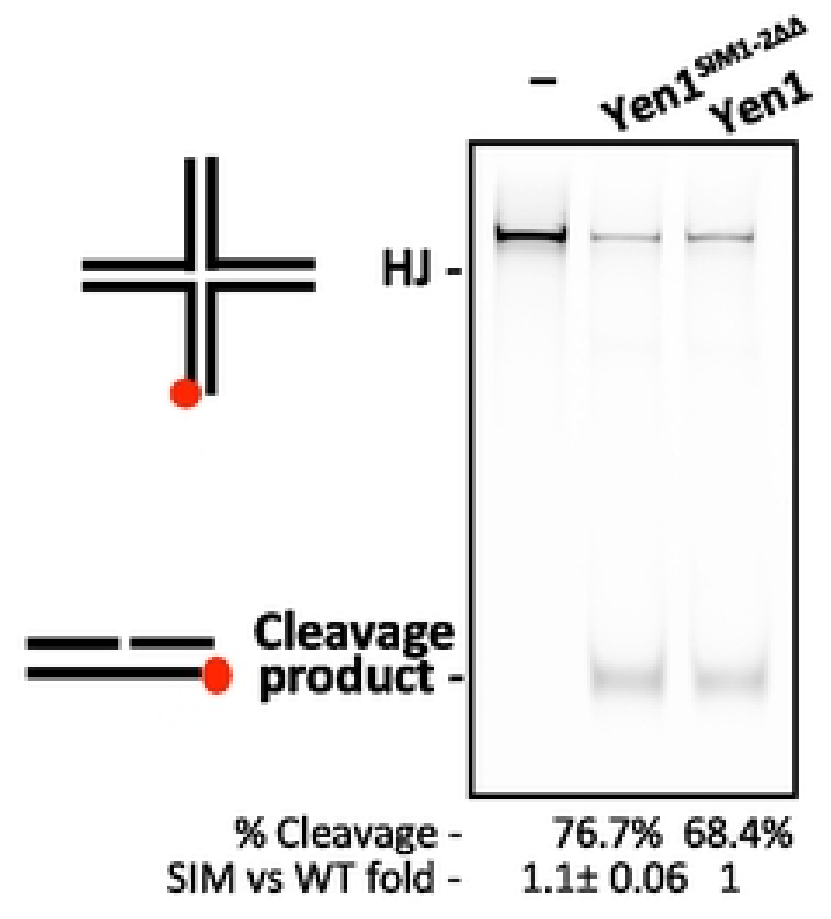

\section{B}

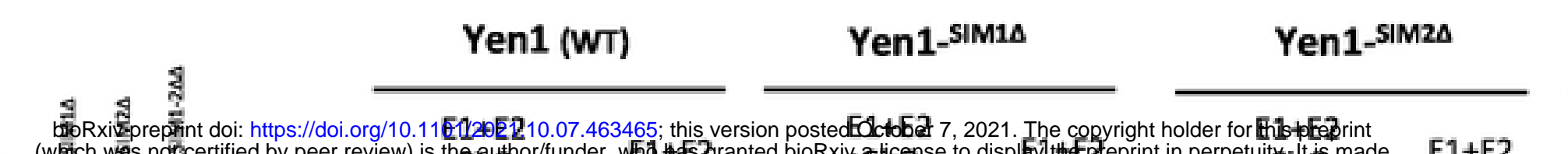

>
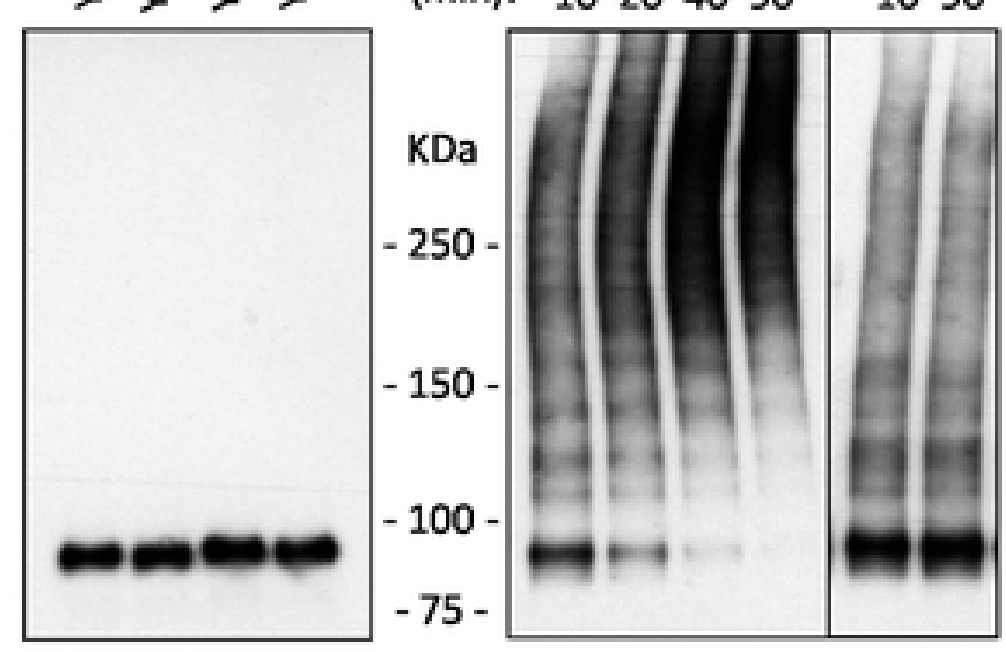

\% Sumo: 44839698

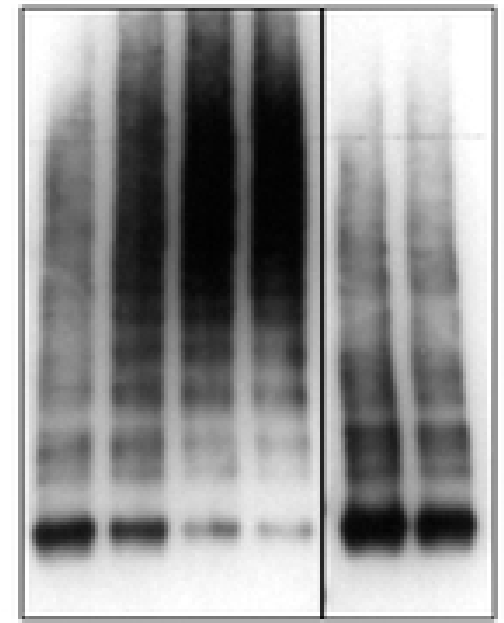

$\overline{10204090} \overline{1090}$

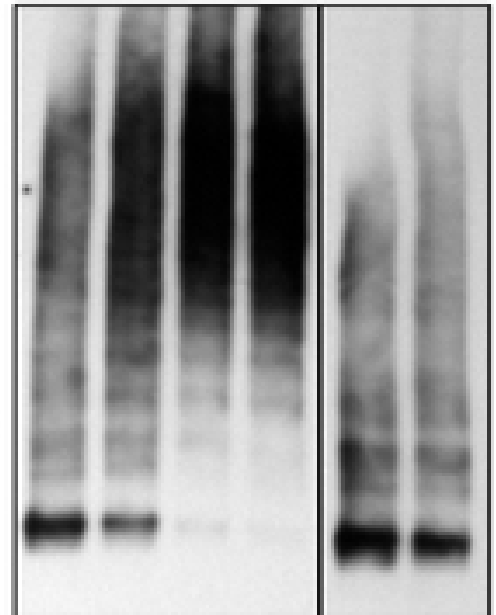

\% Unmd: $\begin{array}{llll}56 & 17 & 4 & 2\end{array}$

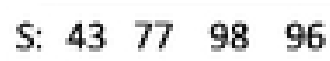

S: $\begin{array}{llll}60 & 79 & 99 & 99\end{array}$

Yen1-SIM1-2MA

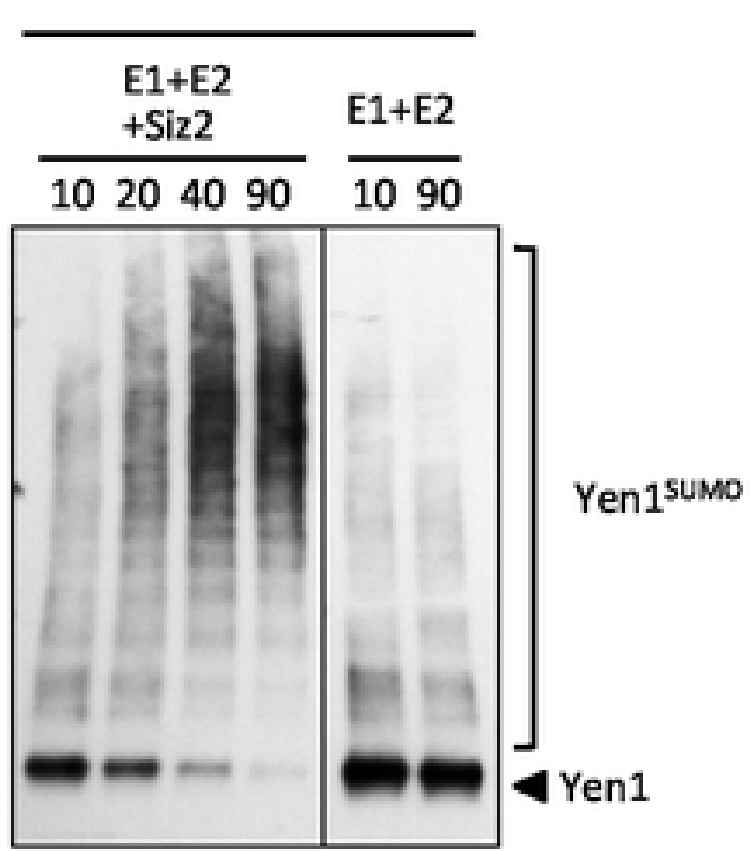

U: $\begin{array}{llll}57 & 23 \quad 2 & 4\end{array}$

U: $\begin{array}{llll}40 & 21 & 2 & 1\end{array}$

S: $\begin{array}{llll}51 & 84 & 96 & 99\end{array}$

U: $\begin{array}{llll}49 & 16 & 4 & 1\end{array}$

Figure 3 
A

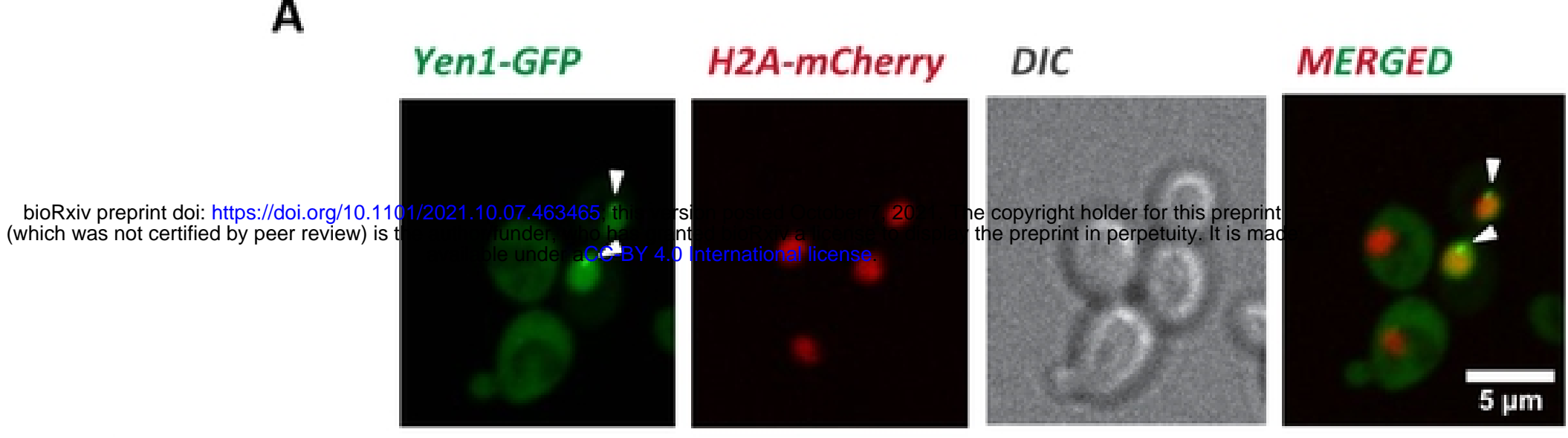

B

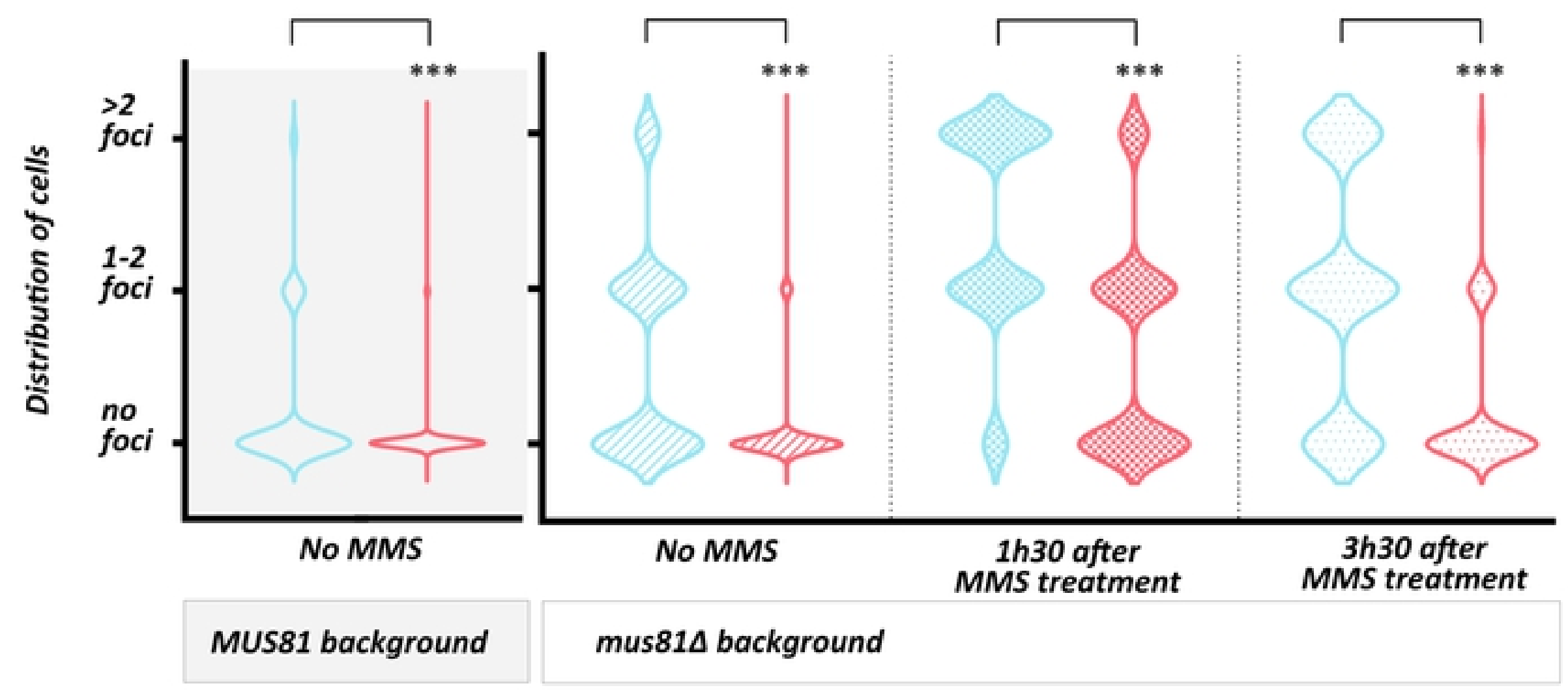

Yen1-GFP variant:

YEN1

yen $1^{\operatorname{sim} 1-2 \Delta \Delta}$

Figure 4 
A

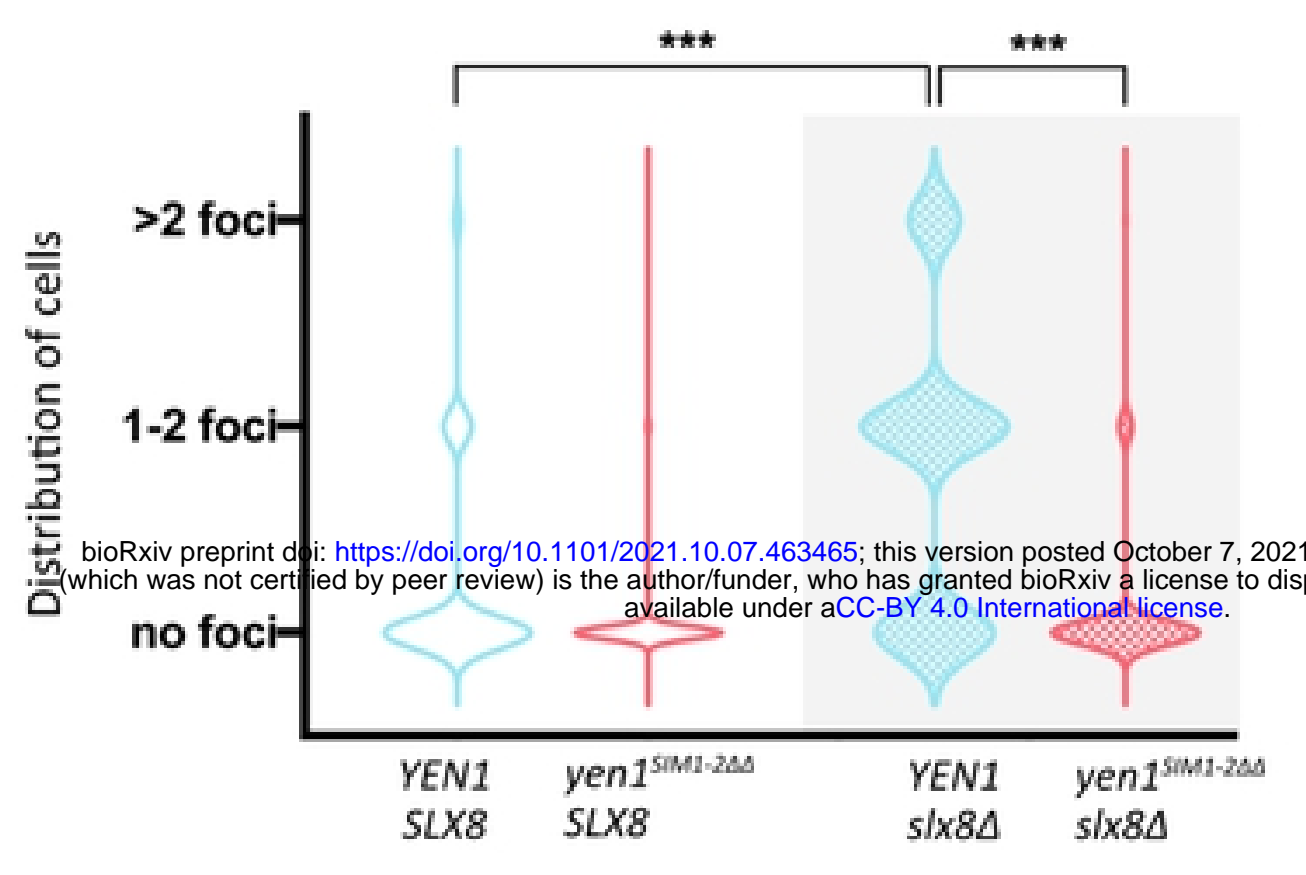

Genetic background

C
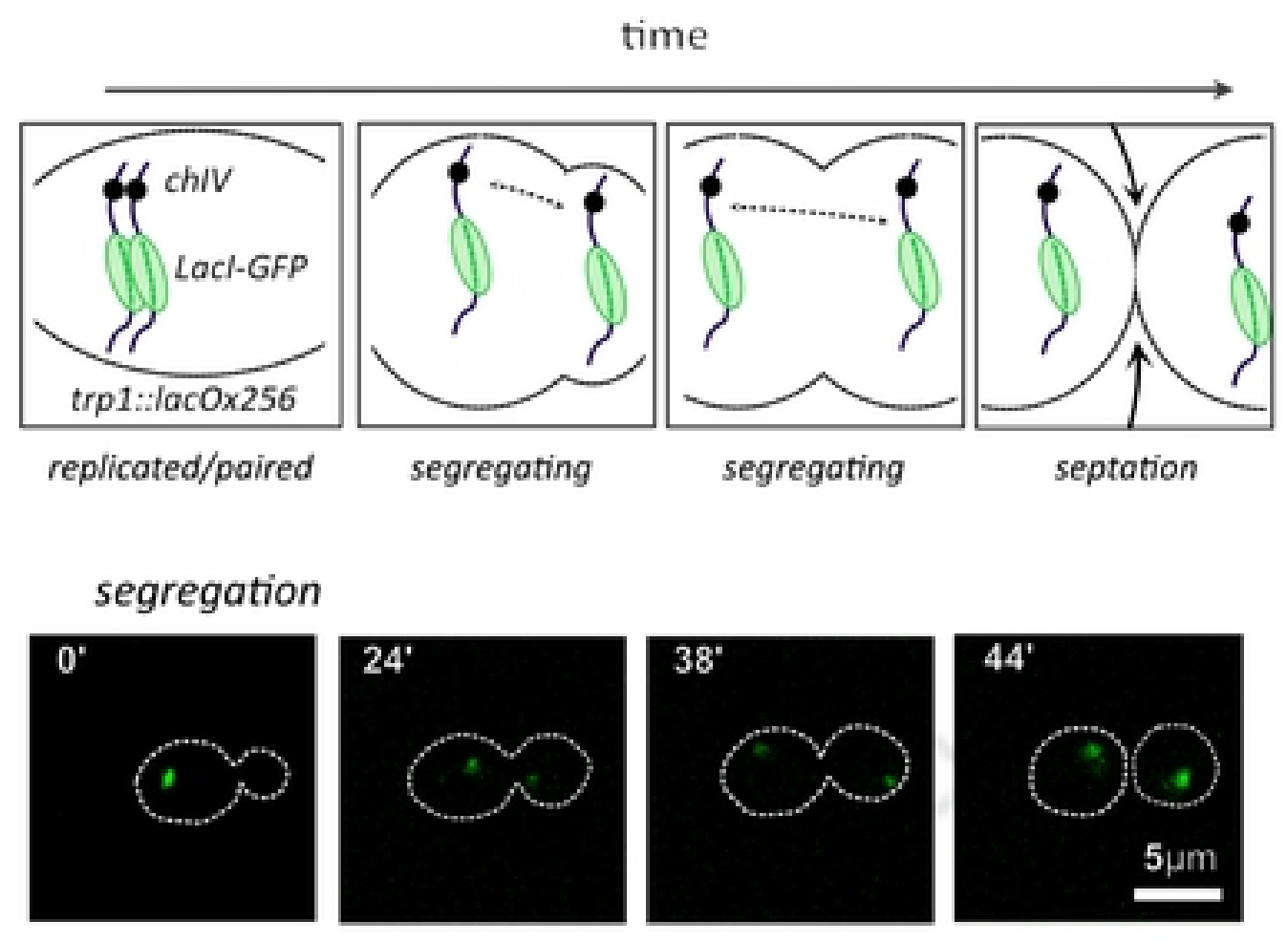

non-disjunction
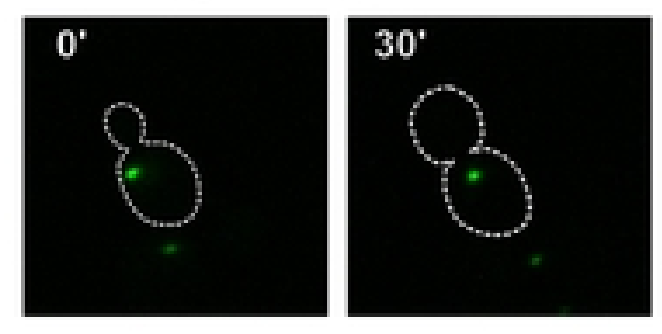

B

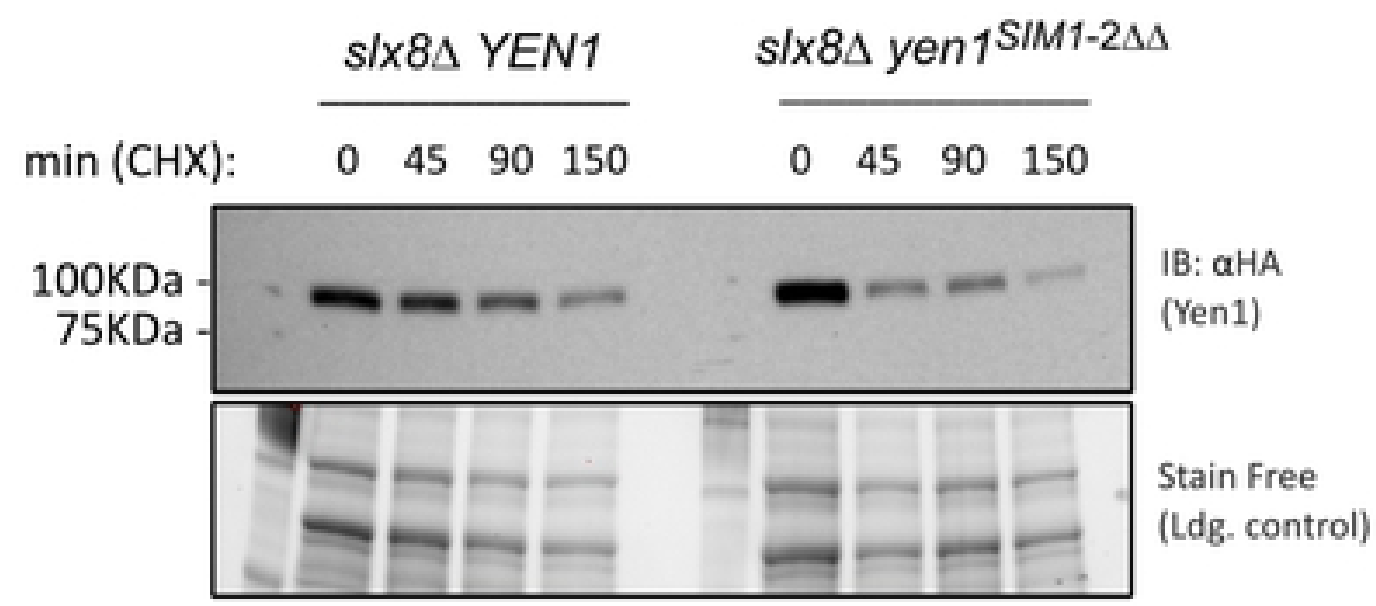

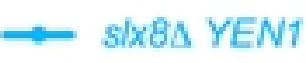

$\multimap$ six8 yen $1^{\text {sIM } 1-2 \Delta \Delta}$

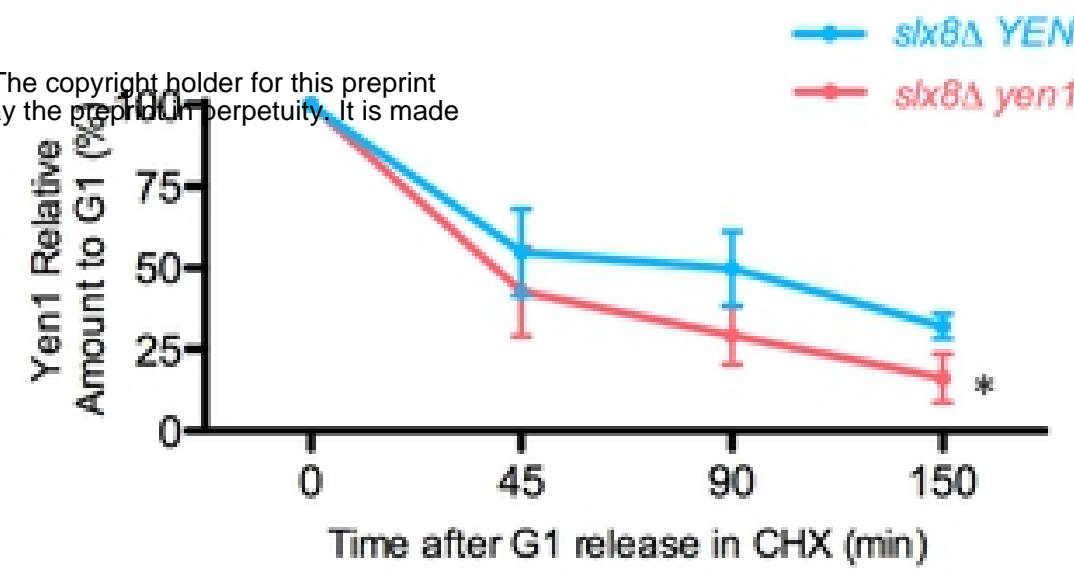

D

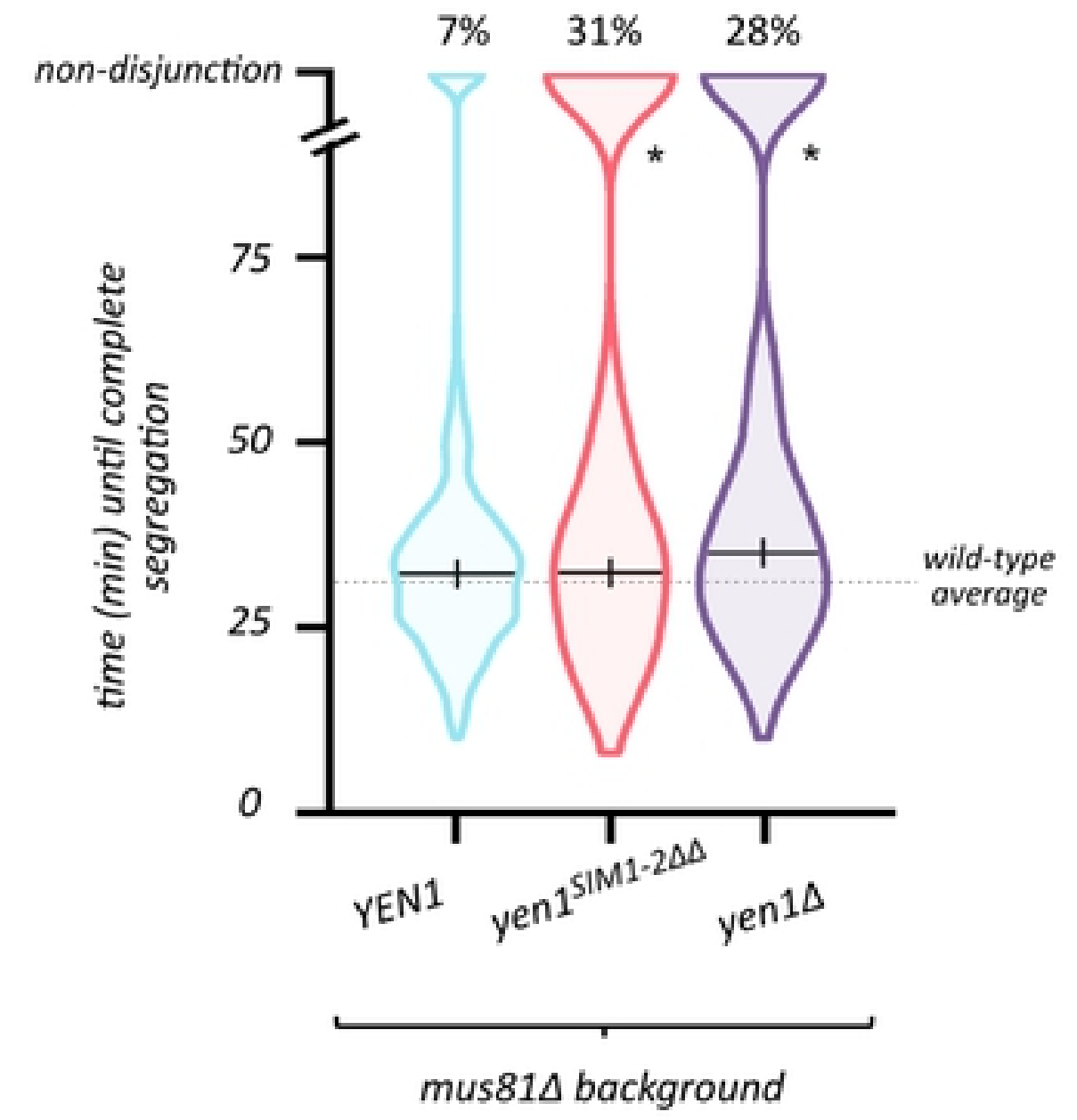

Figure 5 
A

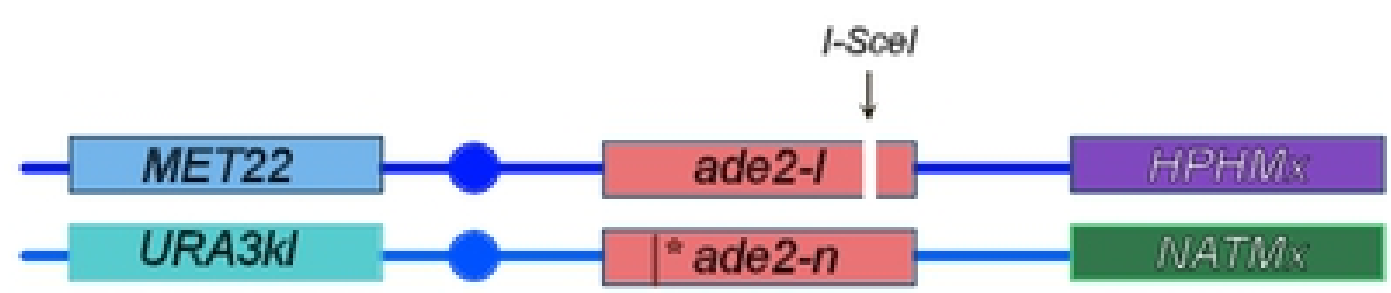

B

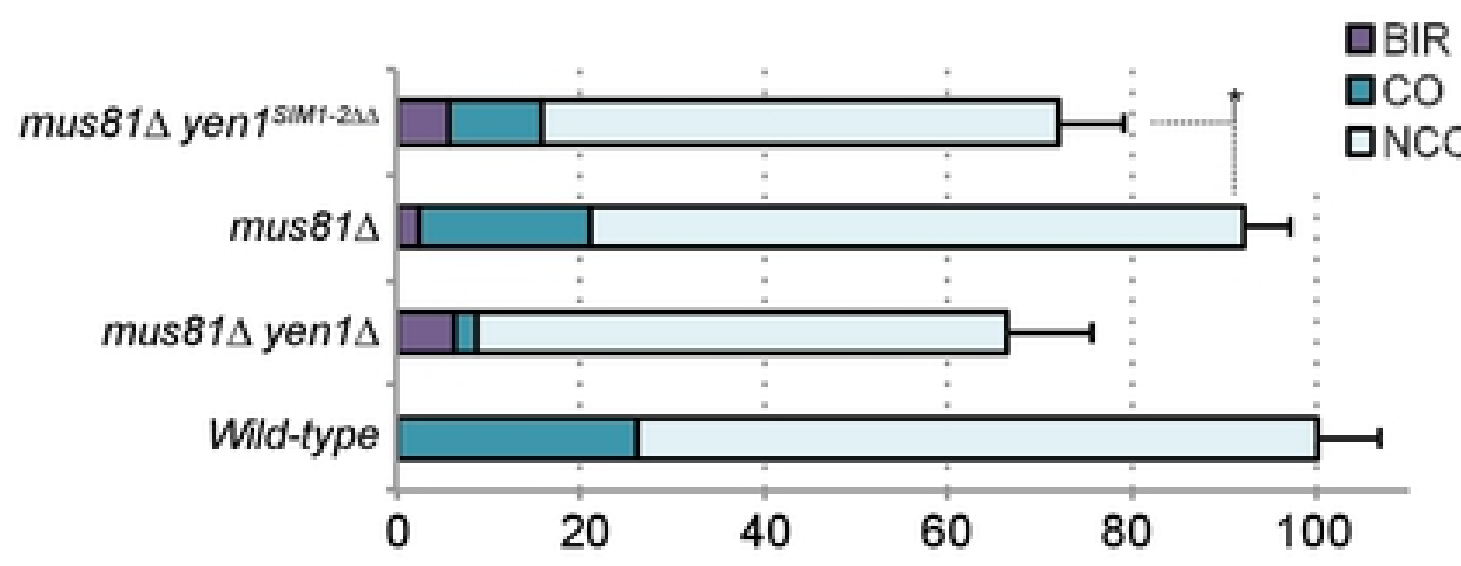

$\%$ Recombinant events (ade2/ADE2 sectored colonies) normalized to PE Gal vs Glu

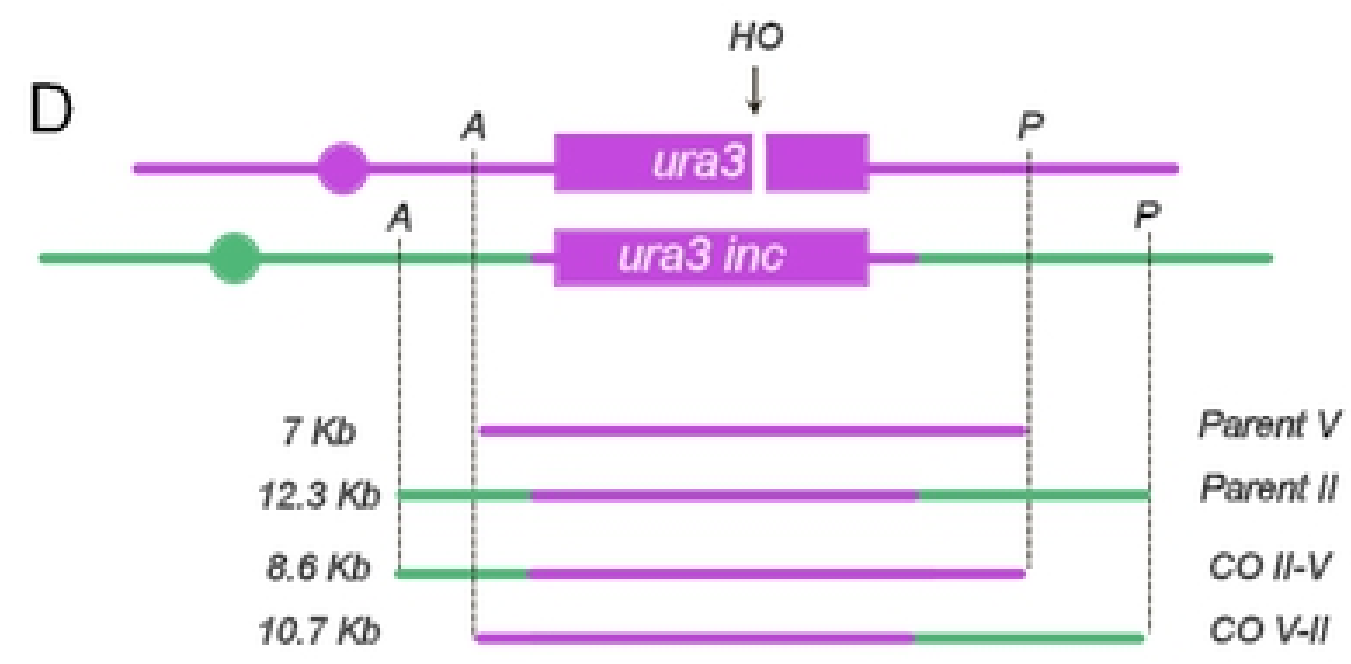

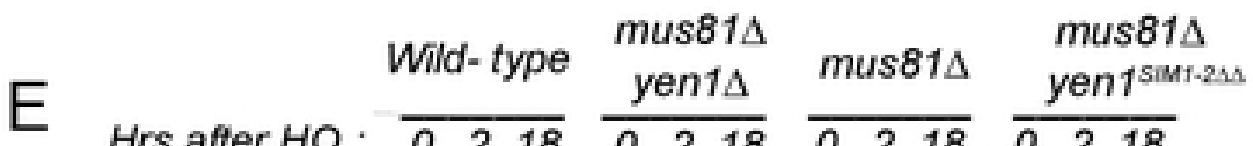

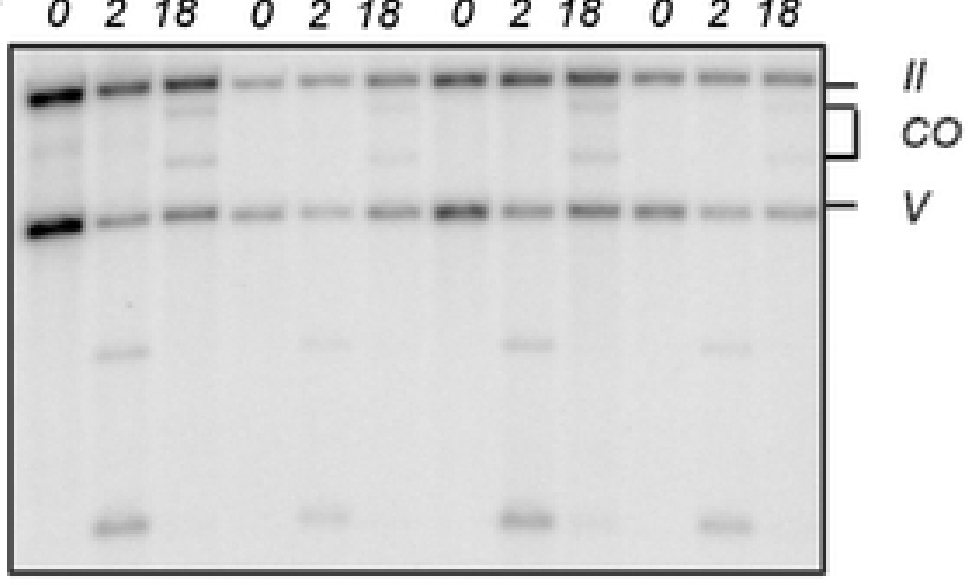

F

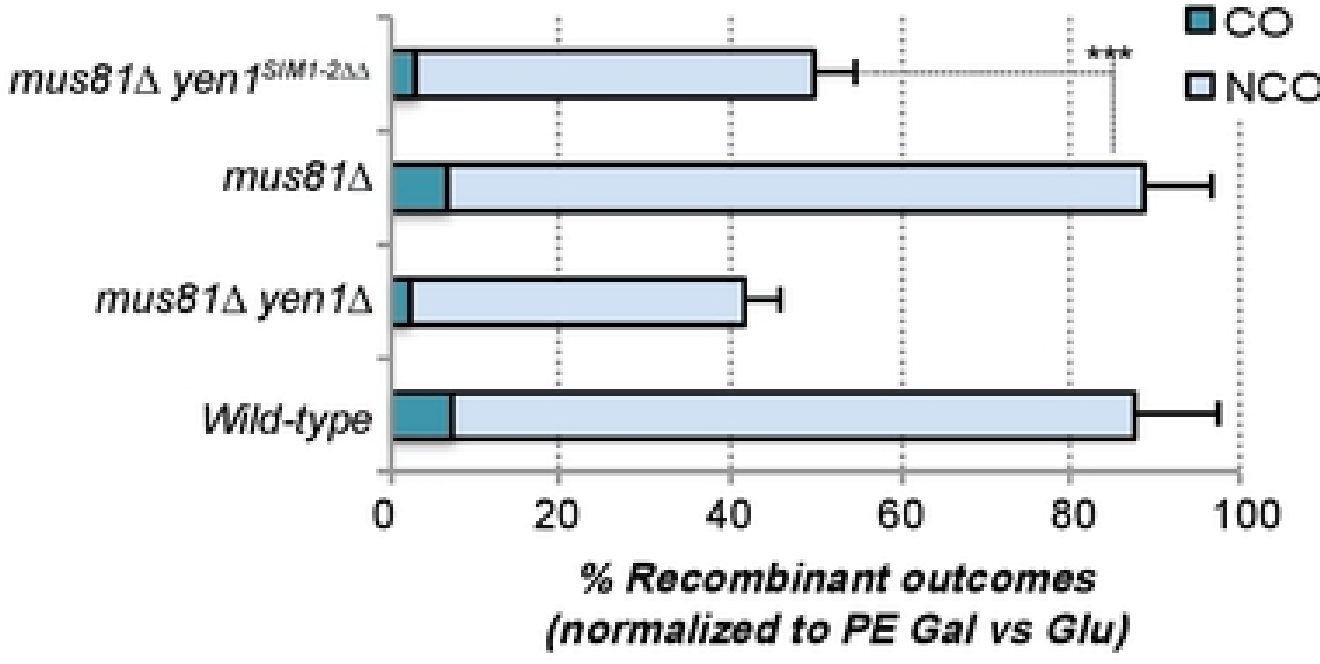

\section{Figure 6}

